\title{
Archéologie du littoral de Guyane. Une région charnière entre les influences culturelles de l'Orénoque et de l'Amazone
}

Stéphen Rostain

\section{Citer ce document / Cite this document :}

Rostain Stéphen. Archéologie du littoral de Guyane. Une région charnière entre les influences culturelles de l'Orénoque et de l'Amazone. In: Journal de la Société des Américanistes. Tome 80, 1994. pp. 9-46;

doi : https://doi.org/10.3406/jsa.1994.1524

https://www.persee.fr/doc/jsa_0037-9174_1994_num_80_1_1524

Fichier pdf généré le 05/05/2018 


\begin{abstract}
Archaeology of Coastal Guiana, a pivotal region between the cultural influences of the Orinoco and the Amazon. This study proposes a synthesis of the development of ancient Ameridian communities of the Guiana coastline. Four cultural complexes may be defined. The Aristé complex was developed in the hills of Ouanary east of Guiana and on the coast of Amapá (Brazil). This complex began circa 350 AD, knew two cultural intrusions and ended circa 1750. The Barbakoeba complex was observed in the extreme western part of Guiana and the eastern part of Surinam, while the Themire complex is present on the central coast of Guiana. These two complexes are dated between 500 and 1600-1650 AD. The Koriabo complex was widely diffused in the Guianas. It began around 1 100-1200 AD in the interior, and $1200-1300$ on the coast, and terminated around 1750.-
\end{abstract}

\title{
Resumen
}

SPA : Guayana francesa! litoral! arqueologia! etnohistoria! complejos culturales

\section{Résumé}

Cette étude analyse les développements des anciennes communautés amérindiennes du littoral de Guyane. Quatre complexes culturels ont été reconnus. Le complexe Aristé s'est développé dans les collines de Ouanary à l'est de la Guyane, et sur le littoral d'Amapa (Brésil). II débuta vers 350 de notre ère, connut deux intrusions culturelles, et s'acheva vers 1750. Le complexe Barbakoeba est repéré à l'extrême ouest de la Guyane et à l'est du Surinam, tandis que le complexe Thémire est reconnu sur la côte centrale de Guyane. Ces deux complexes sont datés entre 500 et 1600-1650 de notre ère. Le complexe Koriabo connut une vaste diffusion dans les Guyanes. II remonterait à 1100-1200 de notre ère dans l'intérieur, et à 1200-1300 sur le littoral, pour s'achever vers 1750 . 


\title{
ARTICLES \\ ARCHÉOLOGIE DU LITTORAL DE GUYANE, UNE REGION CHARNIÈRE ENTRE LES INFLUENCES CULTURELLES DE L'ORÉNOQUE ET DE L'AMAZONE
}

\author{
Stéphen ROSTAIN *
}

\begin{abstract}
Cette étude analyse les développements des anciennes communautés amérindiennes du littoral de Guyane. Quatre complexes culturels ont été reconnus. Le complexe Aristé s'est développé dans les collines de Ouanary à l'est de la Guyane, et sur le littoral d'Amapá (Brésil). Il débuta vers 350 de notre ère, connut deux intrusions culturelles, et s'acheva vers 1750. Le complexe Barbakoeba est repéré à l'extrême ouest de la Guyane et à l'est du Surinam, tandis que le complexe Thémire est reconnu sur la côte centrale de Guyane. Ces deux complexes sont datés entre 500 et 1600-1650 de notre ère. Le complexe Koriabo connut une vaste diffusion dans les Guyanes. Il remonterait à 1100-1200 de notre ère dans l'intérieur, et à 1200-1300 sur le littoral, pour s'achever vers 1750 .
\end{abstract}

Mots CLÉs : Guyane française, littoral, archéologie, ethnohistoire, complexes culturels.

Archaeology of Coastal Guiana, a pivotal region between the cultural influences of the Orinoco and the Amazon.

This study proposes a synthesis of the development of ancient Ameridian communities of the Guiana coastline. Four cultural complexes may be defined. The Ariste complex was developed in the hills of Ouanary east of Guiana and on the coast of Amapá (Brazil). This complex began circa $350 \mathrm{AD}$, knew two cultural intrusions and ended circa 1750. The Barbakoeba complex was observed in the extreme western part of Guiana and the eastern part of Surinam, while the Themire complex is present on the central coast of Guiana. These two complexes are dated between 500 and 1600-1650 AD. The Koriabo complex was widely diffused in the Guianas. It began around 1100-1200 AD in the interior, and 1200-1300 on the coast, and terminated around 1750 .

KEY wORDS : French Guiana, coast, archaeology, ethnohistory, cultural complexes.

* C.R.A.P., Université de Paris I, 3, rue Michelet, 75006 Paris.

Journal de la Société des Américanistes 1994, 80 : p. 9 à 46. Copyright @), Société des Américanistes. 
Arqueología del litoral de la Guayana francesa. Region de articulación entre las influencias culturales del Orinoco y del Amazonas.

Este estudio propone una síntesis del desarrollo de las antiguas comunidades amerindias del litoral de la Guayana. Se pueden identificar cuatro complejos culturales principales. El complejo Aristé se ha desarrollado en las colinas de Ouanary, al este de la Guayana, y en el litoral de Amapá (Brasil). Aristé comienza en el año 350 de nuestra era, aproximadamente, conoce dos intrusiones culturales y termina alrededor de 1750. El complejo Barbakoeba ha sido localizado en el extremo oeste de Guayana y al este de Surinam, mientras que el complejo Thémire se ha encontrado en la costa central de Guayana. Estos dos complejos han sido fechados entre 500 y 1600-1650 de nuestra era. El complejo Koriabo tuvo una gran difusión en Guayana. En el interior remonta a 1100-1200 de nuestra era y en el litoral a 1200-1300. Este complejo acaba alrededor del año 1750.

Palabras Claves : Guayana francesa, litoral, arqueología, etnohistoria, complejos culturales.

\section{INTRODUCTION}

La préhistoire de la Guyane française était pratiquement inconnue jusqu'à ces dernières années, bien que quelques rares travaux eussent montré qu'il existait un potentiel d'étude important dans cette région. La problématique d'une recherche archéologique en Guyane était de ce fait évidente. Par son unité géographique et humaine, le littoral de Guyane était la région idéale pour amorcer une étude d'intérêt local, connectée à une vision générale de l'archéologie de la côte des autres Guyanes (Amapá, Guyana, Surinam, Vénézuela oriental) ${ }^{1}$.

Durant la recherche, nous avons fouillé ou prospecté 55 sites archéologiques sur le littoral, auxquels il faut ajouter l'étude des champs drainés ainsi que l'analyse de 88 sites prospectés par d'autres équipes. Les sites d'habitat en plein-air apparaissent dans quatre environnements principaux : rivage, cordon sableux quaternaire, berge de fleuve, colline. Des petits sites en abri-sous-roche ont également été occasionnellement occupés par les Amérindiens. Cinq types de sites spécialisés sont distingués d'après leurs fonctions : funéraires, pétroglyphes et ensembles mégalithiques, ateliers de polissoirs, champs drainés, collines à fossé périphérique.

Les vestiges archéologiques étudiés proviennent essentiellement de nos fouilles archéologiques et, dans une moindre mesure, de collections publiques et privées.

- L'étude de 23206 tessons et de 296 poteries entières a permis la définition de sept types et de trois catégories céramiques, ainsi que l'identification d'un autre type ${ }^{2}$.

- Les 4464 pièces lithiques représentent quatre techniques d'obtention, regroupant 31 types d'outils.

- Enfin, divers artefacts amérindiens de coquillage, de résine, de bois, ainsi que des objets européens de traite en verre ou en métal ${ }^{3}$, furent analysés. 
La recherche a mis en évidence la variété culturelle préhistorique du littoral de Guyane, avec la reconnaissance de quatre complexes culturels : Aristé, Thémire et Barbakoeba, Koriabo.

\section{PROPOSITIONS DE DATATIONS DES TRADITIONS CÉRAMIQUES DU LITTORAL DE GUYANE}

\begin{tabular}{|l|l|l|l|l|}
\hline \multicolumn{1}{|c|}{ datation AD } & \multicolumn{1}{|c|}{ tradition } & \multicolumn{1}{c|}{ complexes } & \multicolumn{1}{c|}{ types céramiques } & \multicolumn{1}{c|}{ localisation } \\
\hline $350-1000$ & Incisée-Ponctuée/Polychrome & Aristé ancien & Ouanary encoché & extrême est \\
\hline $700-1400(?)$ & Incisée-Ponctuée & Aristé moyen & Caripo kwep & extrême est \\
\hline $1000-1750$ & Polychrome & Aristé récent & Enfer polychrome & extrême est \\
\hline $1300-1650$ & Arauquinoïde/Polychrome & Thémire & $\begin{array}{l}\text { Cayenne peint } \\
\text { Mahury incisé } \\
\text { Melchior kwep }\end{array}$ & centre et ouest \\
\hline $1000-1400$ & Arauquinoïde & Barbakoeba & Barbakoeba & extrême ouest \\
\hline $1200-1750$ & Incisée-Ponctuée & $\begin{array}{l}\text { Koriabo } \\
\text { Chaton fantastique } \\
\text { Melchior kwep }\end{array}$ & fleuves \\
\hline
\end{tabular}

\section{I. - LES PREMIERS OCCUPANTS}

Des sites pré-céramiques sont connus dans l'intérieur du Surinam et du Guyana, et il doit en exister également en Guyane. Vers 10000 ans avant notre ère, des chasseurs de mégafaune vivaient dans les savanes de Sipaliwini au Surinam (A. Boomert, 1980). En revanche, aucun site pré-céramique n'est actuellement repéré sur le littoral de Guyane, du Surinam, ou d'Amapá, mais il est vrai que les traces de populations pré-céramiques sont difficiles à retrouver. Par ailleurs, si nous attribuons aux groupes céramistes l'ensemble des pièces de quartz taillé fournies par presque tous les sites de Guyane, il demeure possible que des sites pré-céramiques aient été réoccupés ultérieurement par des céramistes, utilisant également des quartz taillés. Les perturbations stratigraphiques peuvent confondre les occupations successives d'un site.

Sur la côte du Maranhão, du Guyana et du Vénézuela, où l'on trouve d'importantes colonies de coquillages, des sites pré-céramiques et céramiques anciens ont été découverts sur des amas coquilliers (complexes Mina, Alaka, Warao). Le plus ancien complexe céramique amazonien actuellement reconnu serait celui de Taperinha, qui a été identifié sur un amas coquillier des environs de Santarém et daté de 5700 à 4300 avant notre ère, et qui présente une céramique non décorée à dégraissant de coquillages broyés, proche de la poterie des complexes Alaka Récent et Mina (A. Roosevelt et al., 1991). Les rares coquillages fréquentant les côtes du Surinam, de Guyane et d'Amapá, ne fournissant pas de quantités suffisantes pour nourrir des groupes de chasseurs/collecteurs, on ne peut donc guère espérer découvrir d'amas coquilliers sur cette partie du littoral. Au Surinam, le plus ancien site céramique est Kaurikreek, daté de 2500-1600 avant notre ère et rattaché à la tradition vénézuélienne Saladoïde ${ }^{4}$ (A. Versteeg, 1978 ; A. Versteeg \& F. Bubberman, 1992). Comme Aad Versteeg (comm. pers., 1993), nous considérons 
comme probable que, durant la longue période archéologiquement inconnue entre 10000 et 2500 avant notre ère, de petites communautés de chasseurs/cueilleurs/collecteurs nomades, laissant peu de traces de leur passage dans le sol, ont vécu dans les forêts de l'intérieur des Guyanes et, sans doute, sur le littoral.

En Guyane, les plus anciens sites céramiques repérés apparaissent à partir du troisième millénaire avant notre ère : La Trinité sur le haut Sinnamary (2490-1870 avant notre ère), Pointe Gravier dans l'Ile de Cayenne (1500 avant notre ère), Topu sur le moyen Sinnamary (1450-1190 avant notre ère) (S. Jérémie et al., 1993 ; P. Nowacki-Breczewski \& O. Puaux, 1992 ; J.-F. Turenne, 1974). Ces datations correspondent à celles du complexe Mina qui s'est développé entre 3000 et 1600 avant notre ère sur la côte du Pará et sur le bas Amazone (M. Simões, 1981). Par ailleurs, en plusieurs endroits de l'Amazonie, des sites céramiques sont datés des alentours de 1000 avant notre ère.

À partir du début de notre ère, plusieurs sites de Guyane furent occupés : Wewe dans le bas Sinnamary (P. Nowacki-Breczewski \& O. Puaux, 1992), Abri Marcel et Carbet Mitan (complexe Aristé) dans le bas Oyapock (S. Rostain, 1994).

\section{II. - LE COMPLEXE ARISTÉ, TRADITIONS INCISÉE-PONCTUÉE ET POLYCHROME}

Le complexe culturel Aristé fut originellement défini sur le littoral central et septentrional d'Amapá par Betty Meggers et Clifford Evans (1957). Il a été ensuite reconnu dans la baie d'Oyapock, en Guyane française (H. Petitjean Roget, 1980). Le territoire Aristé s'étendait sur la côte ${ }^{5}$, de la rivière Araguari aux Monts de l'Observatoire au moins, soit approximativement $370 \mathrm{~km}$ de longueur pour 10 à $100 \mathrm{~km}$ de largeur (Figure 1).

\section{1. - Composantes du complexe Aristé}

\section{A - Les sites d'habitat}

Treize sites d'habitat sont repérés sur le littoral d'Amapá (B. Meggers \& C. Evans, 1957), pour 22 en Guyane (Figure $1 \&$ annexe 1). Le complexe Aristé des collines de Ouanary est caractérisé par deux modèles d'habitat : une unité de base, rassemblant la communauté dans un village en plein-air, et des unités restreintes en abri-sous-roche ${ }^{6}$, où l'espace domestique individuel du village était reconstitué temporairement.

Les sites communautaires en plein-air, localisés sur les collines ou les berges des cours d'eau, s'étendent sur 2000 à $40000 \mathrm{~m}^{2}$. Ces sites présentent un dépôt réparti de façon relativement uniforme, la couche archéologique atteignant 8 à $70 \mathrm{~cm}$ de profondeur. On peut distinguer des petits sites pouvant abriter de 40 à 200 personnes environ et des installations nettement plus vastes pouvant loger de 500 à 4000 individus. Les villages du bas Oyapock, décrits dans les archives des $\mathrm{XVI}^{\mathrm{e}}$ et $\mathrm{XVII}^{\mathrm{e}}$ siècles - à l'époque où le complexe Aristé dominait encore la région - étaient apparemment constitués de plusieurs maisons familiales. 


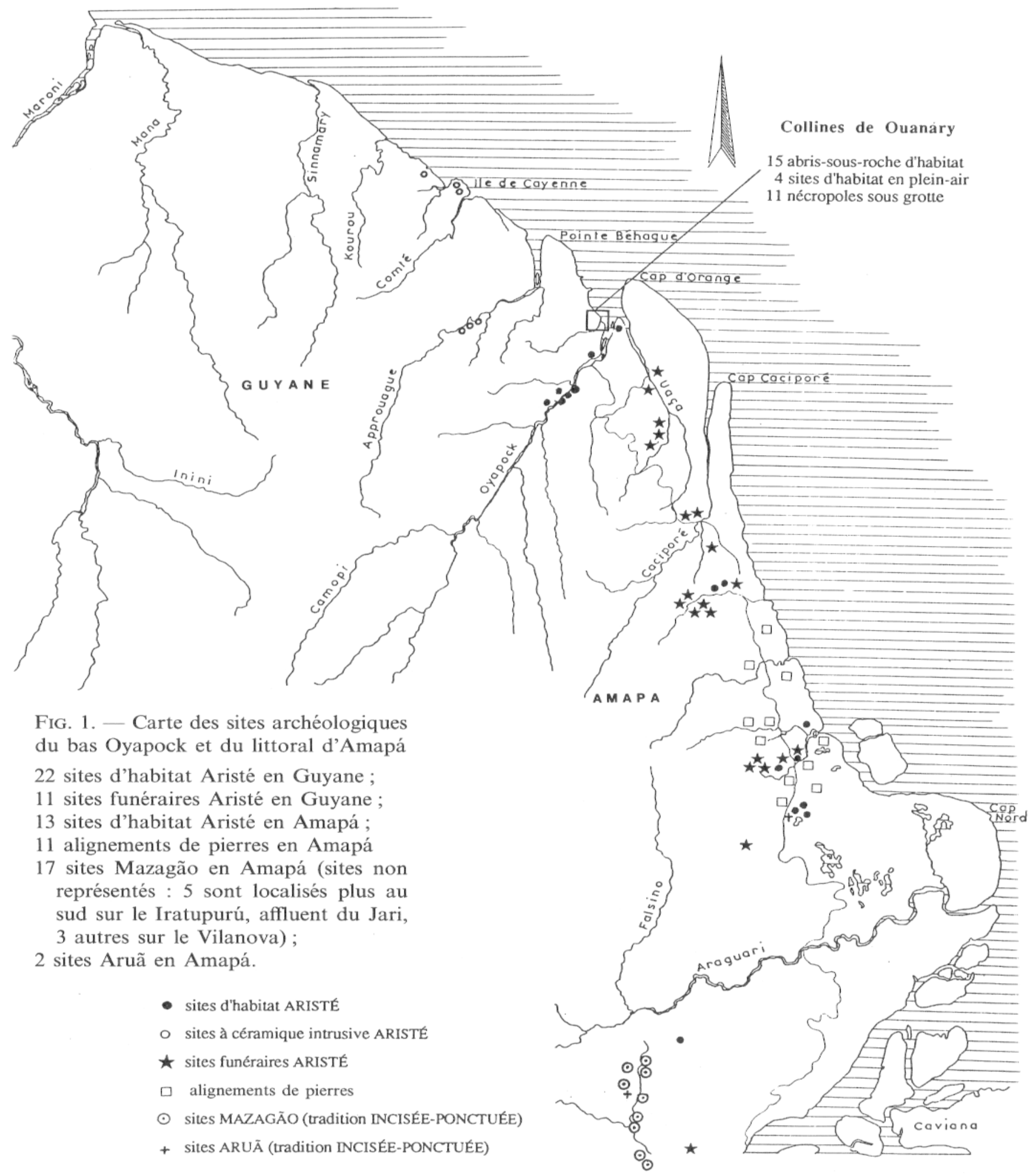


Les sites en abri-sous-roche sont caractérisés par de petites dimensions, d'une moyenne de $28 \mathrm{~m}^{2}$, et présentent généralement un replat devant l'entrée, autorisant une plus grande surface d'occupation. Les fouilles ont montré l'absence de stratification dans le matériau, produit d'altération de la roche, qui tapisse la base des cavernes. Les vestiges peuvent être retrouvés jusqu'à la roche-mère, apparaissant entre 30 et $80 \mathrm{~cm}$ de profondeur. Les fouilles ont montré que ces abris semblent avoir été temporairement occupés à plusieurs reprises, par un nombre restreint de personnes, tandis que les unités résidentielles principales du groupe étaient les villages en plein-air. Il est possible que ces abris-sous-roche aient été utilisés à l'occasion de retraites, comme celles encore observées dans certains groupes amérindiens actuels, où la vie est ponctuée de périodes d'isolement : rites de puberté des garçons et des filles, réclusion des apprentis chamanes, initiation des chefs, deuils, isolement pour certaines activités artisanales comme le travail de la pierre ou la fabrication de la poterie, etc.

Cette dualité de l'habitat Aristé, communautaire et individuel, reflète une structuration de la société, encore observable dans certains groupes amérindiens actuels.

\section{$B$ - Les sites funéraires}

Les 21 sites funéraires d'Amapá sont pour la plupart localisés sur des élévations naturelles, et non loin d'une rivière (B. Meggers \& C. Evans, 1957). Les 11 sites funéraires Aristé des collines de Ouanary sont des nécropoles sous grotte, présentant des sépultures secondaires en urnes. Le sous-complexe Aristé Ancien serait plutôt caractérisé par la sépulture secondaire en urne après décharnement, qui fut apparemment remplacée par la crémation et la sépulture en urne cinéraire durant le sous-complexe Aristé Récent (B. Meggers \& C. Evans, 1957).

Les nécropoles Aristé sont classées en quatre modèles spécifiques :

- Les nécropoles en plein-air où les urnes sont alignées à même le sol.

- Les nécropoles enterrées où les urnes sont enterrées en ligne, à faible profondeur.

- Les nécropoles sous grotte où, disposées contre la paroi rocheuse, les urnes sont nombreuses, jusqu'à 85 pièces pouvant être retrouvées dans une même grotte (de 1 à 23 urnes par site en Guyane).

- Les nécropoles en puits artificiel - le seul mesuré atteint $2,5 \mathrm{~m}$ de profondeur pour 1,2 $\mathrm{m}$ de diamètre - sont fermées par une dalle de granite. Ces puits furent creusés pour remédier à l'absence de grottes dans certains endroits d'Amapà. Les urnes sont disposées sur le sol nivelé d'une chambre latérale.

\section{C - Les ensembles mégalithiques}

Les 11 ensembles mégalithiques connus sur la côte centrale d'Amapá sont localisés pour la plupart sur des élévations naturelles offrant un vaste panorama. Ils présentent des compositions de dalles de granite verticales, disposées en rangée, en cercle grossier ou en triangle. Ces sites, à fonction vraisemblablement 
cérémonielle, furent attribués au complexe Aruã (B. Meggers \& C. Evans, 1957), mais cette identification fut récemment mise en doute (A. Boomert, 1986). En effet, la localisation des ensembles mégalithiques et le matériel céramique retrouvé indiqueraient plutôt qu'ils étaient Aristé.

Ces ensembles mégalithiques étaient peut-être liés à des cultes similaires à ceux que rendaient, au $\mathbf{X V I I}^{\mathrm{e}}$ siècle, les Arauakí, les Tapajó et les Trombeta du moyen Amazone, à des idoles de bois ou de pierre (rites de fertilité, de mariage, de parturition, etc.) (E. Nordenskiöld, 1930).

\section{D - La céramique}

La céramique du complexe Aristé d'Amapá a été classée en deux types ordinaires et cinq types décorés (B. Meggers \& C. Evans, 1957). Bien que le matériel Aristé de Guyane, tessons et pièces entières, soit très proche de celui d'Amapá, les différences existantes ont justifié la définition de types indépendants.

Le sous-complexe Aristé Ancien présente une céramique rattachée à la tradition Incisée-Ponctuée, qui coexiste, en fin de séquence, avec une composante Polychrome. Le type Ouanary encoché (3981 tessons et 38 poteries entières) est caractérisé par un dégraissant de sable quartzeux, une pâte de couleur principalement brun-rougeâtre ${ }^{7}$. Les formes domestiques sont des plaques, des plats creux, des jattes et des bols, et les formes funéraires sont des jarres, des tablettes, des jattes et des coupelles. Le décor, présent sur $10 \%$ du matériel, est constitué de peinture rouge ou blanche, d'incisions simples, d'encoches sur les lèvres et les bords (Figure 2).

Le sous-complexe Aristé Moyen voit l'apparition de nouvelles céramiques, également rattachées à la tradition Incisée-Ponctuée. Le type Caripo kwep (1 669 tessons et 11 poteries entières) est caractérisé par un dégraissant d'écorces brûlées et broyées ${ }^{8}$, une pâte de couleur principalement jaune rougeâtre. Les formes domestiques sont des plaques, des plats creux, des assiettes et des pots, et les formes funéraires sont des jarres, des écuelles et des plaques. Le décor, présent sur $15 \%$ du matériel, est surtout fait d'incisions répétitives en droites simples ou en motifs complexes, parfois peints (Figure 3).

Le sous-complexe Aristé Récent est complètement rattaché à la tradition Polychrome. Le type Enfer polychrome ( 7718 tessons et 111 poteries entières) est caractérisé par un dégraissant de chamotte et une couleur de pâte gris sombre à gris très sombre. Les formes domestiques sont des plaques, des plats creux, des jattes, des pots et des bols, et les formes funéraires sont des jarres, des tablettes, des jattes et des plats creux très variés. Le décor peint polychrome, présent sur $22 \%$ du matériel, est dominé par les courbes et les spirales (Figure 4).

La catégorie céramique Hocco fer (50 tessons) apparaît uniquement dans l'abri-sous-roche de Carbet Mitan.

\section{E- La pierre}

Le matériel lithique demeure relativement rare dans les sites d'Amapá, car cette région est pauvre en gisements de roche, et il est possible que des outils de bois ou d'os aient suppléé au manque d'outils de pierre. 

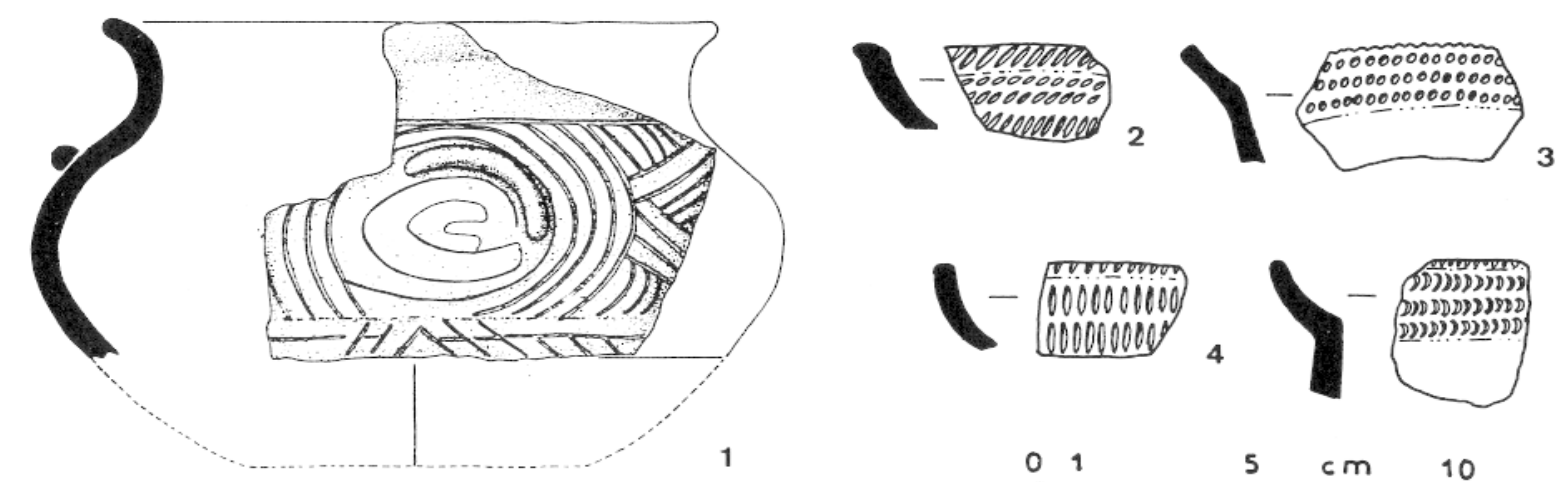

5

1
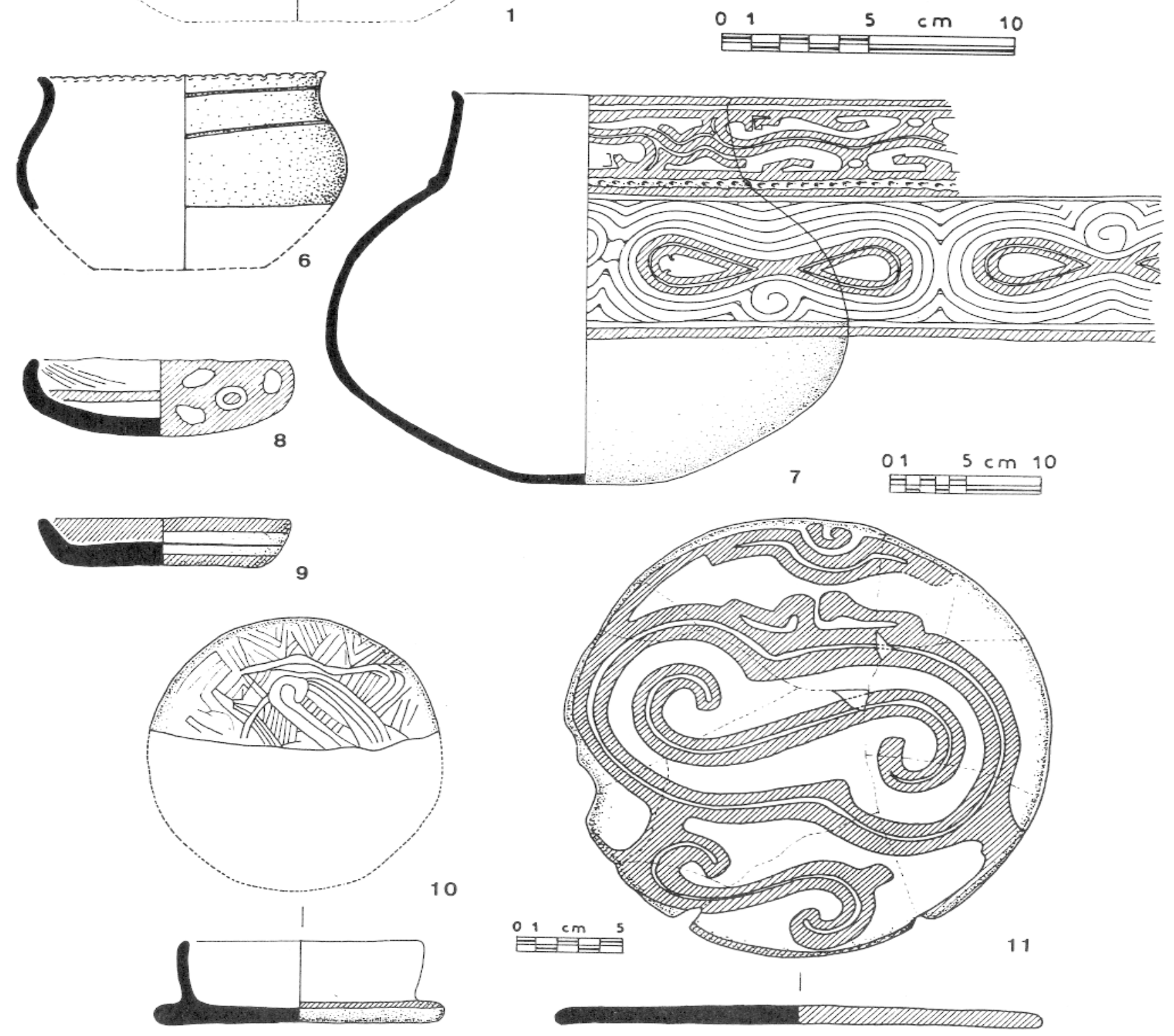

Fig. 2. - Céramiques du complexe Aristé Ancien

1) Bol incisé et à cordon appliqué, site Abri Marcel. 2) Bord intérieur encoché, Abri Marcel. 3) Bord intérieur ponctué et lèvre encochée, Abri Marcel. 4) Bord intérieur et lèvre encochés, Abri Marcel. 5) Bord intérieur et lèvre encochés, Abri Marcel. Échelle différente : 6) Jatte funéraire à lèvre encochée, Trou Reliquaire. 7) Urne globulaire à décor peint en rouge (hachures obliques) sur fond blanc (sans pointillés) et cordon horizontal imprimé sur l'encolure, Grotte Geay. Échelle différente : 8) Coupelle peinte en blanc et rouge, Trou Reliquaire. 9) Assiette peinte en rouge, Trou Agae. 10) Coupelle (tablette) à rebord peint en rouge et blanc, et face inférieure incisée, Trou Reliquaire. 11) Plaque peinte en rouge, Trou Agae. 

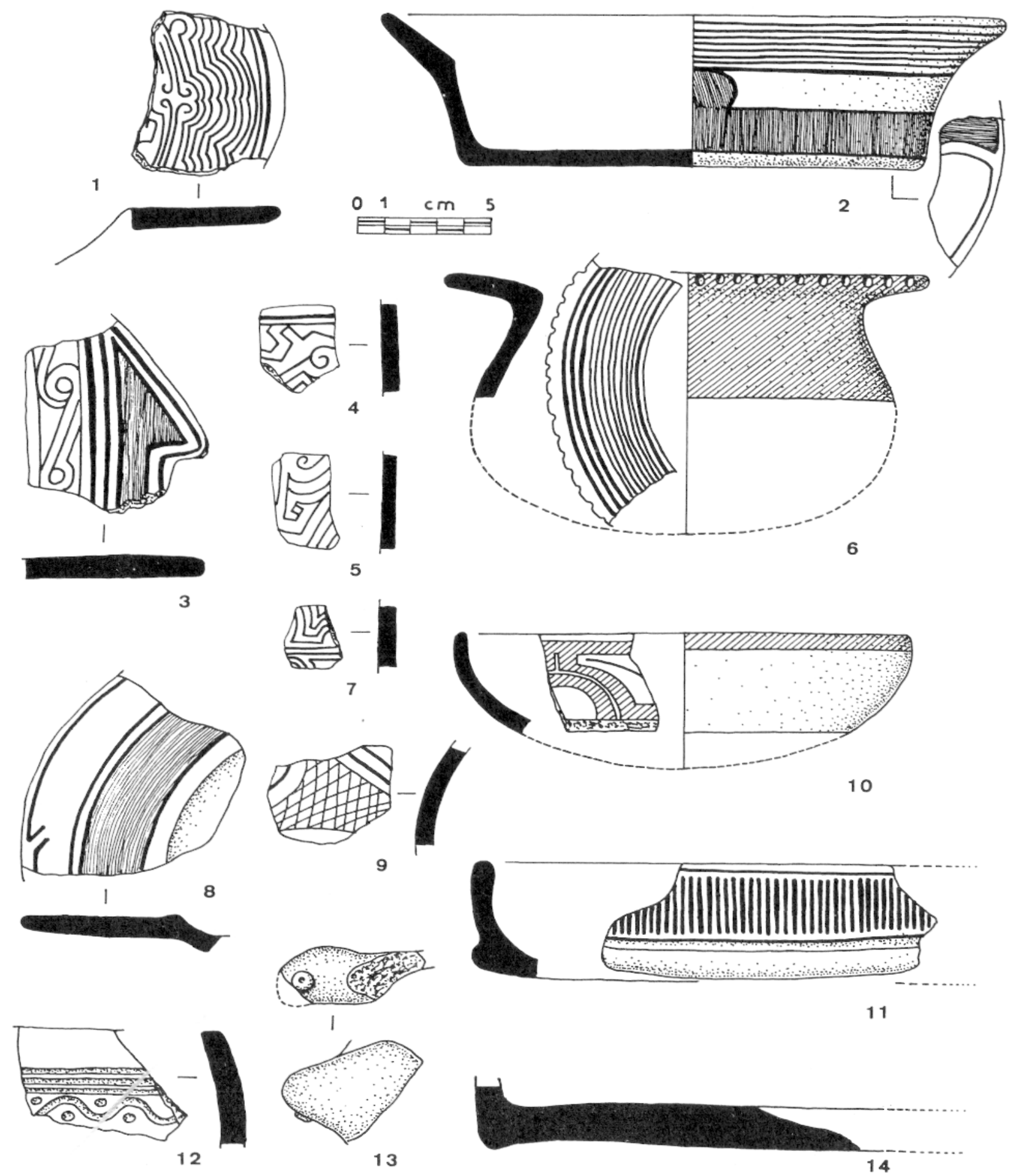

Fig. 3. - Céramiques du complexe Aristé Moyen

1) Bord horizontal incisé, Moustique. 2) Plat creux à décor incisé et peigné, avec une vue de la face inférieure, Moustique. 3) Bord horizontal incisé, Moustique. 4) Tesson incisé, Moustique. 5) Tesson incisé, Moustique. 6) Pot incisé et peint à l'extérieur, Moustique. 7) Tesson incisé, Moustique. 8) Bord horizontal incisé, Moustique. 9) Tesson incisé, Moustique. 10) Écuelle funéraire peinte en rouge et blanc, Trou Agae. 11) Plat creux incisé, Moustique. 12) Bord légèrement évasé incisé, Moustique. 13) Modelé zoomorphe. Moustique. 14) Plaque à cuire funéraire, à rebord peint en rouge à l'extérieur et à motifs peints sur la lacs inférieure, Trou Agae. 

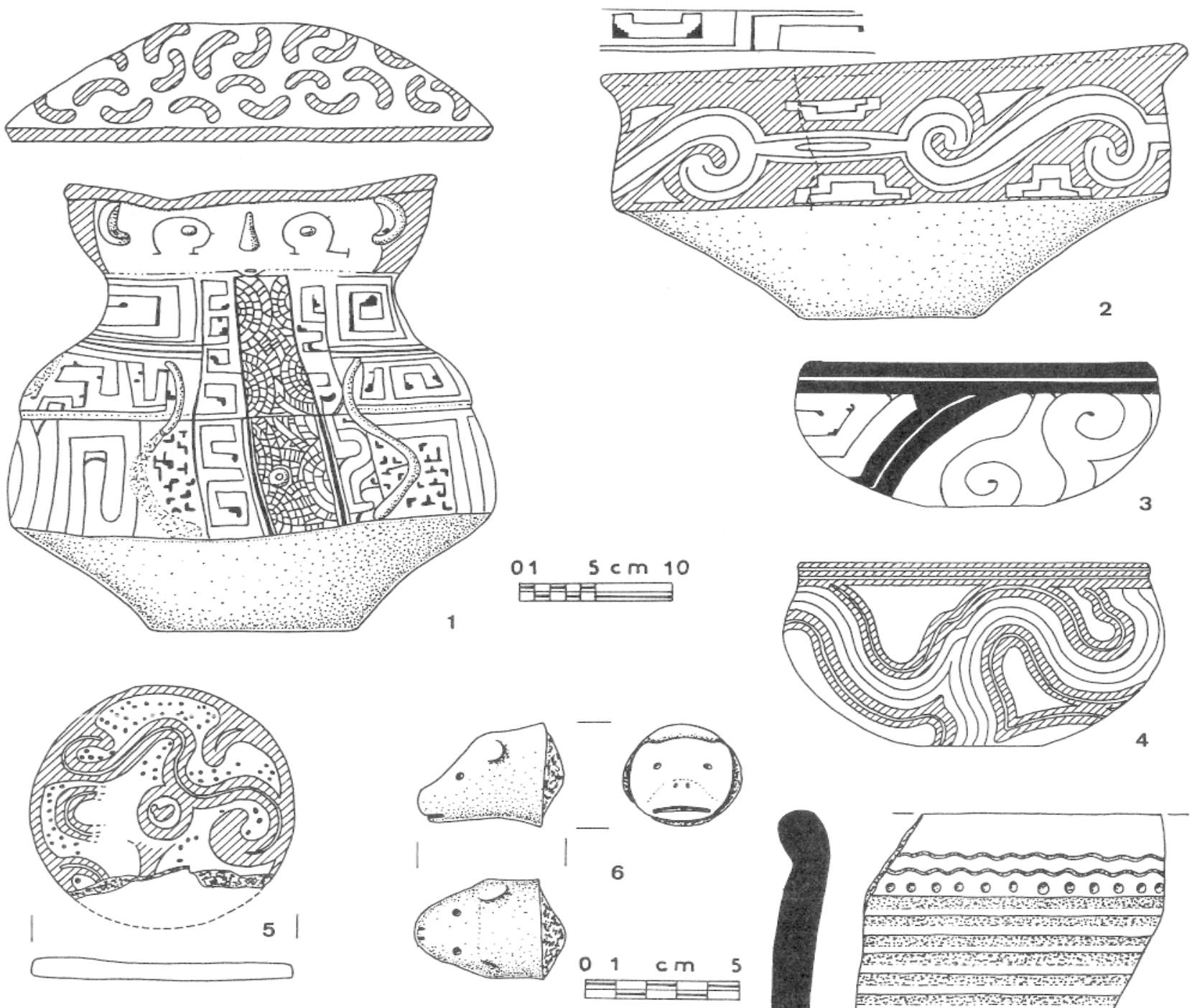

3
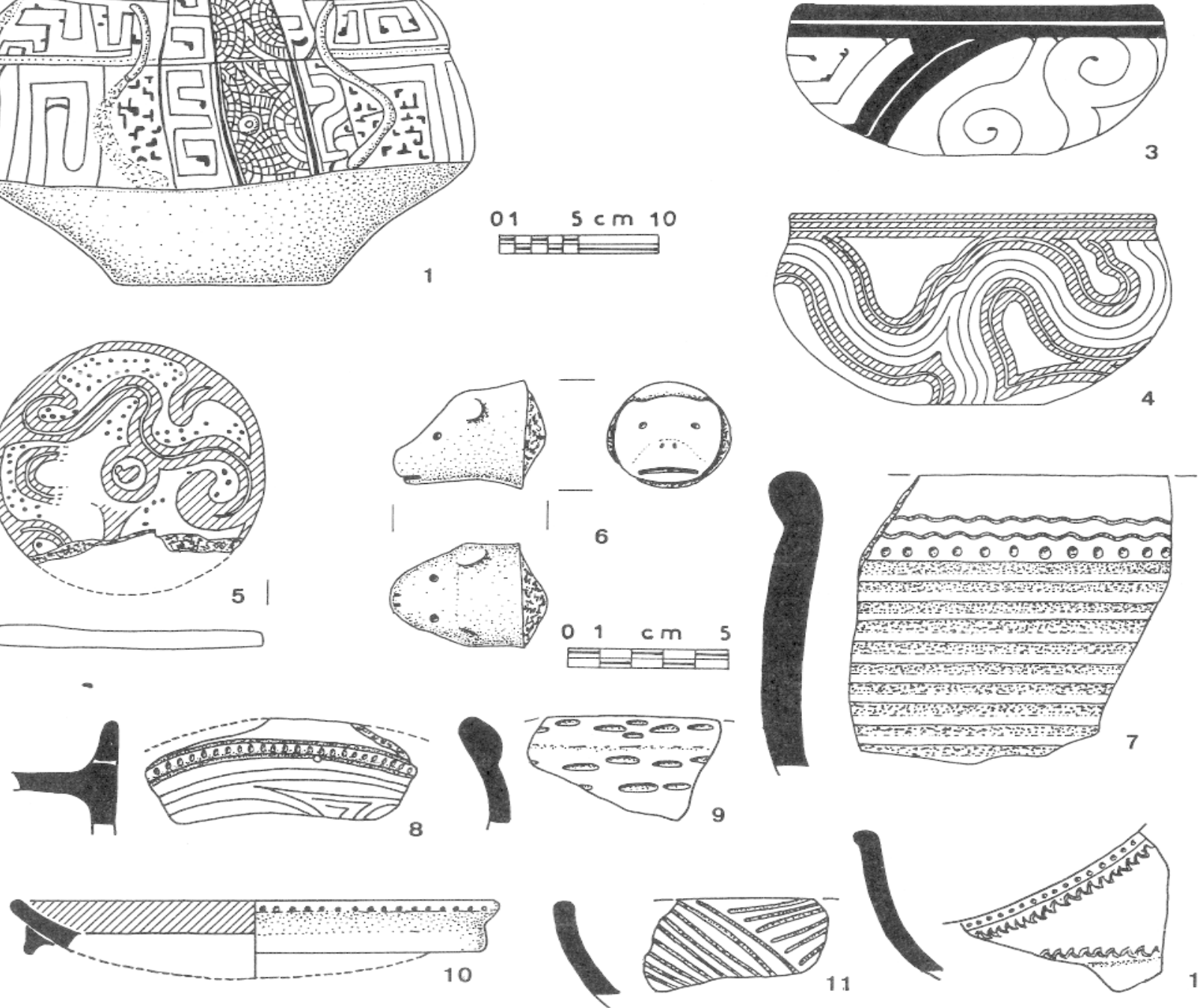

Fig. 4. - Céramiques du complexe Aristé Récent

1) Jarre funéraire à profil composite, munie de son couvercle, à décor peint en rouge sur fond blanc et à modelés anthropomorphes simples appliqués, Gros Montagne. 2) Bassin à décor peint en rouge sur fond blanc, Gros Montagne. 3) Jatte à décor peint en brun foncé sur fond blanc, Grotte Geay. 4) Jatte à décor peint en rouge sur fond blanc, Grotte Geay. 5) Plaque à décor peint en rouge, Trou Agae. 6) Modelé zoomorphe (loutre ?), Enfer. 7) Bord incisé à l'extérieur, Abri Patagaïe. 8) Bord de tablette à face inférieure incisée, Abri Patagaïe. 9) Bord encoché à l'intérieur, Moustique. 10) Assiette à rebord, peinte et lèvre encochée, Abri Patagaïe. 11) Bord incisé à l'extérieur, Gaston. 12) Bord lobé incisé à l'extérieur, Abri Marcel. 
Dans les collines de Ouanary, les quartz taillés ${ }^{9}$ et les outils non façonnés dominent largement dans les sites d'habitat. Les nombreux déchets de taille, percuteurs et enclumes découverts attestent que les quartz étaient débités sur place. Les outils taillés sont des grattoirs, des racloirs et des dents de râpe. Des pièces esquillées, ayant pu servir d'outils, ont été également découvertes. Les outils non façonnés ont eu des usages divers (Figure 5) : débitage du quartz (percuteur, enclume, enclume/percuteur), polissage et aiguisage d'outils (polissoir), ouverture de fruits durs et de graines (casse-noix), finition de surface des poteries (lissoir), broyage (meule et molette). La pierre polie est rare, représentée par quelques lames de hache de différentes formes et un petit ciseau. La présence de lames inachevées dans les abris-sous-roche suggère qu'elles pouvaient être polies sur place, à l'aide de polissoirs portatifs.

L'outillage de pierre est nettement plus rare dans les grottes funéraires. On y trouve parfois quelques quartz taillés, des outils non façonnés et de rares pièces polies (Figure 5), peut-être déposés avec les biens du défunt. Découverts dans le site de Trou Reliquaire, une palette et un broyeur à pigments présentant des traces de colorant rouge semblent indiquer que des préparations colorantes, vraisemblablement destinées à enduire les ossements lors de sépultures secondaires, pouvaient être effectuées sur place.

\section{$E$ - Les artefacts européens}

Des objets de troc européens ont été trouvés dans neuf nécropoles d'Amapá et quatre de Guyane ; ce sont, essentiellement, des perles de verre, des morceaux de verre (ayant peut-être appartenu à des verres à boire), des clous, des couteaux, des bagues, des faïences, des clochettes, des médailles, un couteau, un miroir, un dé percé et une machette.

\section{2 - Situation du complexe Aristé dans les Guyanes}

\section{A - Les datations}

Le complexe Aristé fut tout d'abord approximativement daté entre le xIII ${ }^{\mathrm{e}}$ et le $\mathrm{XVII}^{\mathrm{e}}$ siècles de notre ère (B. Meggers \& C. Evans, 1957). A l'époque des fouilles en Amapá, les datations au ${ }^{14} \mathrm{C}$ n'existant pas, le placement chronométrique du début du complexe avait fait l'objet de simples suppositions.

Les seules datations absolues du complexe Aristé ont été obtenues dans deux abris-sous-roche d'habitat et deux grottes funéraires des collines de Ouanary. Les âges fournis vieillissent considérablement le début jusqu'alors supposé de ce complexe : il commencerait en Guyane vers 350 de notre ère ( $c f$. annexe) ${ }^{10}$.

Dans les quatre nécropoles sous grotte de Guyane ayant fourni des objets de troc européens, les perles de verre, fondues et parfois amalgamées avec des ossements humains brûlés, indiquent que le défunt pouvait être paré d'ornements de valeur lors de sa crémation. Ces données confirment que les cimetières Aristé étaient encore utilisés à l'époque historique, à partir de 1500. Les perles retrouvées 

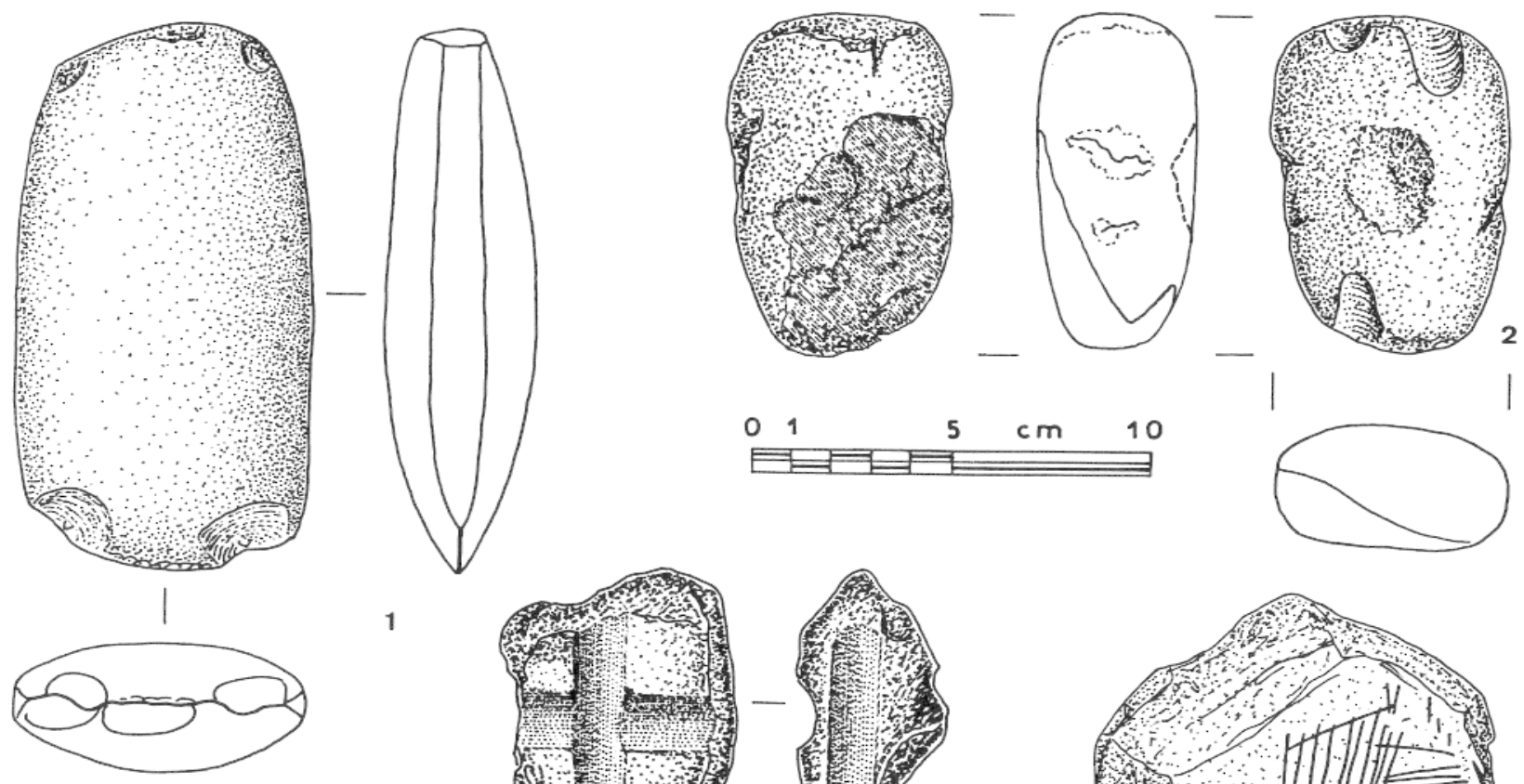

1
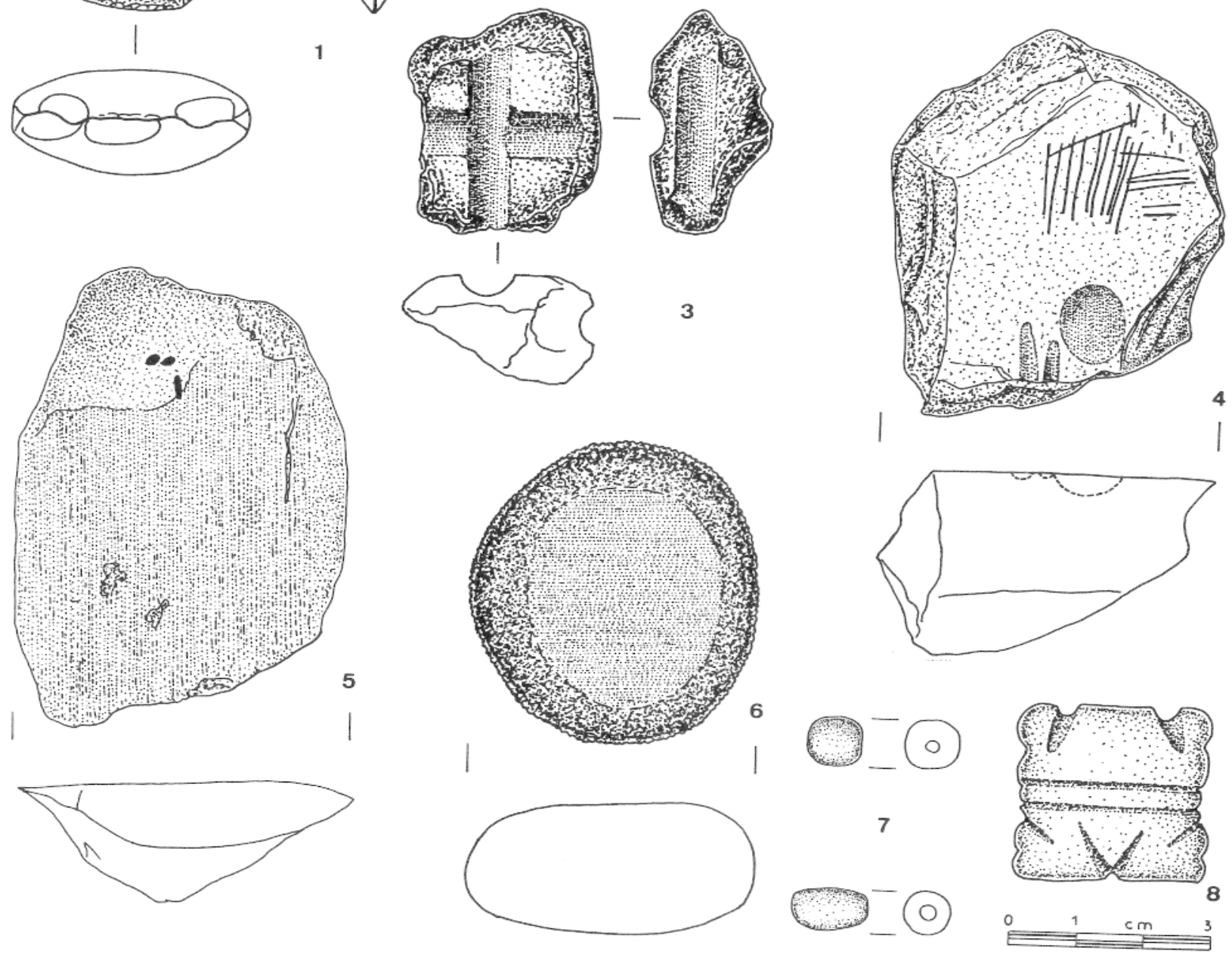

FIG. 5. - Outils de pierre de divers sites de Guyane

1) Lame de hache simple de tuf présentant deux fausses coches au tranchant, Bois Diable (complexe Thémire). 2) Enclume/percuteur sur galet de schiste vert avec un négatif d'enlèvement couvrant une face, Carbet Mitan (complexe Aristé). 3) Polissoir à trois gouttières d'amphibolite litée, Kamuyune (complexe Thémire). 4) Polissoir complexe sur dalle de schiste andésitique avec deux rainures, des rayures rectilignes et une cupule de polissage, Manioc (complexe Aristé). 5) Polissoir-aiguisoir sur dalle de schiste andésitique avec une face plane polie par l'usage, présentant trois taches de pigment rouge, Abri Patagaïe (complexe Aristé). 6) Molette avec une face finement polie par l'usage, Glycérias (complexe Thémire). Échelle différente : 7) Perles de quartz poli, Trou Agae (complexe Aristé). 8) Pendentif de pierre verte polie en forme de batracien, plage de Montravel, Ile de Cayenne. 
ne sont toutefois pas suffisamment caractéristiques pour être datées d'une époque précise.

Des précisions chronologiques sont apportées en revanche par les faïences découvertes dans les nécropoles de Trou Delft et Trou Reliquaire (Y. Le Roux, comm. pers., 1993 ; H. Petitjean Roget, 1993). Ces pièces proviennent probablement d'ateliers hollandais, où la production commerciale d'écuelles, comme celle de Trou Delft, débuta à la fin du XVII ${ }^{\mathrm{e}}$ siècle, tandis que les faïences de Trou Reliquaire sont datées de 1730-1750 (Y. Le Roux, comm. pers., 1993). Ces faïences furent peut-être échangées avec des navigateurs français ${ }^{11}$, ou ont simplement pu être récoltées par les Amérindiens sur des installations coloniales abandonnées.

La présence de faïences datées dans des nécropoles Aristé prouve que ces sites étaient encore utilisés au milieu du $\mathrm{XVIII}^{\mathrm{e}}$ siècle. Par ailleurs, les urnes de section rectangulaire de type Enfer polychrome, découvertes dans ces sites, indiquent l'émergence d'influences nouvelles dans ce complexe. Il est probable que ces nécropoles d'époque coloniale représentent les dernières manifestations du complexe Aristé, en cours de fusionnement avec des complexes exogènes.

\section{$B-E ́$ volution du complexe Aristé}

Les plus anciennes populations céramiques d'Amapá actuellement connues appartiennent au sous-complexe Aristé Ancien.

Se fondant sur l'étroite ressemblance technique et stylistique entre les types anciens des complexes Aristé et Mazagão (ce dernier apparaissant dans le sud de l'Amapá), Betty Meggers et Clifford Evans (1957) postulaient une base commune, antérieure à l'arrivée des deux complexes en Amapá, et localisaient cet ancêtre dans le bas Amazone plutôt que dans les Guyanes.

D'un autre côté, une appartenance totale du complexe Aristé à la tradition Polychrome (A. Boomert, 1986 ; B. Meggers \& C. Evans, 1957) est infirmée par les récentes découvertes archéologiques. On observe en effet une transformation progressive de ce complexe depuis la tradition Incisée-Ponctuée (Aristé Ancien) vers la tradition Polychrome (Aristé Récent). Ce rattachement du sous-complexe Aristé Ancien à la tradition Incisée-Ponctuée situerait son origine sur le moyen Amazone, principal foyer de cette tradition. L'appartenance des complexes Mazagão et Aristé Ancien à la tradition Incisée-Ponctuée justifie ainsi leur origine commune et explique les convergences, soupçonnées par Arie Boomert (1986), entre ces types et le complexe Koriabo, également rattaché à cette tradition. Les complexes de tradition Incisée-Ponctuée sont généralement datés entre 900 et 1600 de notre ère (P. Hilbert, 1968 ; A. Prous, 1986; M. Simões, 1983), mais l'ancienneté des datations du sous-complexe Aristé Ancien nous incite à vieillir d'autant cette tradition.

Après leur implantation en Amapá, les complexes Mazagão et Aristé Ancien suivirent des développements indépendants. Le complexe Mazagão, qui demeure rattaché à la tradition Incisée-Ponctuée, se maintint dans le sud de la région, et le complexe Aristé occupa la région au nord de l'Araguari (Figure 1). Les deux complexes suivirent alors des évolutions différenciées, tout en conservant des relations d'échange. 
La constance stylistique de la céramique Aristé est symptomatique d'une culture stable et persistante, ayant subi peu d'influences extérieures après la période initiale d'adaptation. Les thèmes décoratifs anciens sont repris et développés tout au long de la séquence, et l'apparition de la polychromie ne modifie pas fondamentalement les décors mais les enrichit en permettant, par la souplesse du traité pictural, des compositions plus complexes.

Hormis la polychromie, la seule intrusion notable dans le complexe Aristé est représentée par le type Caripo kwep ${ }^{12}$. Celui-ci, clairement rattaché à la tradition Incisée-Ponctuée, présente plusieurs similitudes de forme et de décor avec la céramique, de même tradition, des complexes Silves et Itacoatiara, sur le moyen Amazone, en aval de la confluence avec le Madeira ${ }^{13}$. Le type Caripo kwep n'apparaît jamais hors des sites Aristé de Guyane, où il est plus ou moins bien représenté (jusqu'à $58 \%$ de la céramique dans la grotte Moustique), mais il est apparemment absent des sites d'Amapá, à l'exception d'une urne funéraire et de son couvercle. Si ce type provient bien du moyen Amazone, son absence sur le littoral d'Amapá indiquerait que sa diffusion suivit une autre voie, peut-être par l'intérieur et l'Oyapock. Les formes et les décors céramiques du type Caripo kwep sont nettement différents de ceux des types Ouanary encoché et Enfer polychrome, à l'exception de l'urne Caripo kwep d'Amapá qui est une imitation claire des urnes Enfer polychrome. Cette copie, ainsi que la présence presque systématique du type Caripo kwep dans les sites Aristé de Ouanary, suggère que les populations porteuses de cette nouvelle céramique entretinrent d'étroites relations avec les communautés locales. Toutefois, l'originalité que conserve la céramique Caripo kwep écarte l'hypothèse d'une fusion ou d'une assimilation totale. Le type Caripo kwep marque le second mouvement de la tradition Incisée-Ponctuée vers l'Amapá, et la présomption d'un foyer commun avec le complexe Aristé serait un des éléments explicatifs de leur cohabitation. La fusion et la scission sont des mécanismes classiques dans les relations à l'intérieur des groupes ou inter-tribales des Guyanes (P. Grenand, 1982 ; D. Schœpf, 1972).

L'absence de travaux archéologiques en Guyane et au Surinam empêchaient Betty Meggers et Clifford Evans (1957) de bien cerner géographiquement le complexe Aristé. Trouvant des similitudes entre les types Aristé et la céramique récoltée sur le littoral central du Surinam, ils proposaient d'étendre le territoire Aristé jusqu'à cette région. Mais depuis, la céramique surinamienne a été classée avec certitude dans les complexes Koriabo, Barbakœba ou Kwatta. Nos travaux ont en outre montré clairement que le complexe Aristé ne s'étendait pas au-delà des collines de Ouanary, à l'exception de quelques rares intrusions sur l'Approuague, l'Ile de Cayenne et, peut-être, la Montagne de Kaw (Figure 1).

L'étendue des sites d'habitat, la forte densité de nécropoles et d'abris-sous-roche occupés, la diversité des innovations céramiques locales semblent indiquer que le bas Oyapock fut un foyer de développement Aristé particulièrement dense. La présence de collines littorales a joué un rôle fixateur auprès des populations, mais aussi la qualité portuaire et stratégique de la baie d'Oyapock, surtout utile pour les bateaux européens. A l'époque historique, les chefs des confédérations pan-tribales d'Amapá habitaient cette région, qui fut probablement un centre politique important. 
Bien que les communautés Aristé aient occupé une région fréquemment visitée par les Européens dès les premiers temps de la Conquête, elles furent moins affectées par ce contact que les autres communautés du bas Amazone. Cette dernière aire, qui a représenté très tôt un enjeu territorial important entre Portugais et autres nations européennes, connut de nombreux conflits. Les inimitiés entre groupes amérindiens s'en trouvèrent exacerbées, et le choc épidémique fut vraisemblablement violent dans l'embouchure de l'Amazone, où la démographie amérindienne chuta plus rapidement que sur la côte des Guyanes (P. \& F. Grenand, 1987). Cela explique la disparition des complexes archéologiques Mazagão et Maraca peu après la Conquête. Au contraire, par leur localisation privilégiée dans le nord de l'Amapá et dans la baie d'Oyapock, les groupes Aristé survécurent plus longtemps à la rencontre.

La Conquête provoqua de grandes migrations, et des populations fuirent les Antilles, le delta de l'Orénoque et le bas Amazone, pour s'installer en Amapá. Ce brassage culturel perturba la stabilité des Aristé. Des regroupements d'ethnies et des fusions de cultures matérielles s'effectuèrent, qui donnèrent naissance à des communautés hybrides reconstruites parmi lesquelles, dans le nord de l'Amapá, les Palikur actuels (groupe linguistique aruak).

\section{III. - LE COMPLEXE BARBAKOEBA, TRADITION ARAUQUINOIDE}

Nous avons repéré le complexe culturel Barbakoeba à l'extrême ouest du littoral de Guyane. Il se rattache aux développements pré-coloniaux tardifs de la tradition Arauquinoïde du littoral oriental du Surinam. Le complexe Barbakoeba a été défini au Surinam par Arie Boomert (1977 \& 1993). Le territoire Barbakoeba s'étendait de la rivière Commewijne, au Surinam, jusqu'au fleuve Mana au moins, soit approximativement $120 \mathrm{~km}$ de longueur par $10 \mathrm{à} 15 \mathrm{~km}$ de largeur (Figure 6), mais des sites de ce complexe seront peut-être découverts dans le futur entre les fleuves Mana et Kourou.

\section{1. - Composantes du complexe Barbakoeba}

Les sept sites connus au Surinam sont localisés sur des cheniers ${ }^{14}$ et présentent un niveau archéologique continu de terra preta ${ }^{15}$, de 30 à $40 \mathrm{~cm}$ d'épaisseur. En Guyane, nous n'avons pour l'instant reconnu le type céramique Barbakœba que dans le site de Crique Jacques ${ }^{16}$. Ce site est localisé sur un chenier du bas Mana et présente une simple fosse remplie de tessons (A. Cornette, 1985). Aucun site Barbakoeba n'est repéré à l'est de Kourou, mais l'absence de données archéologiques sur le littoral entre le Mana et la Karouabo empêche pour l'instant de tracer les limites exactes de l'extension du complexe Barbakoeba en Guyane.

De très grands ensembles de champs drainés sont localisés dans les marais au sud des cheniers présentant les sites d'habitat. Les champs surélevés, d'une hauteur variant entre 50 et $150 \mathrm{~cm}$, sont de forme carrée $(4 \times 4 \mathrm{~m})$ à rectangulaire $(3-4 \times$ $10 \mathrm{~m}$ environ). Ils sont parfois associés à des canaux étroits, disposés perpendi- 


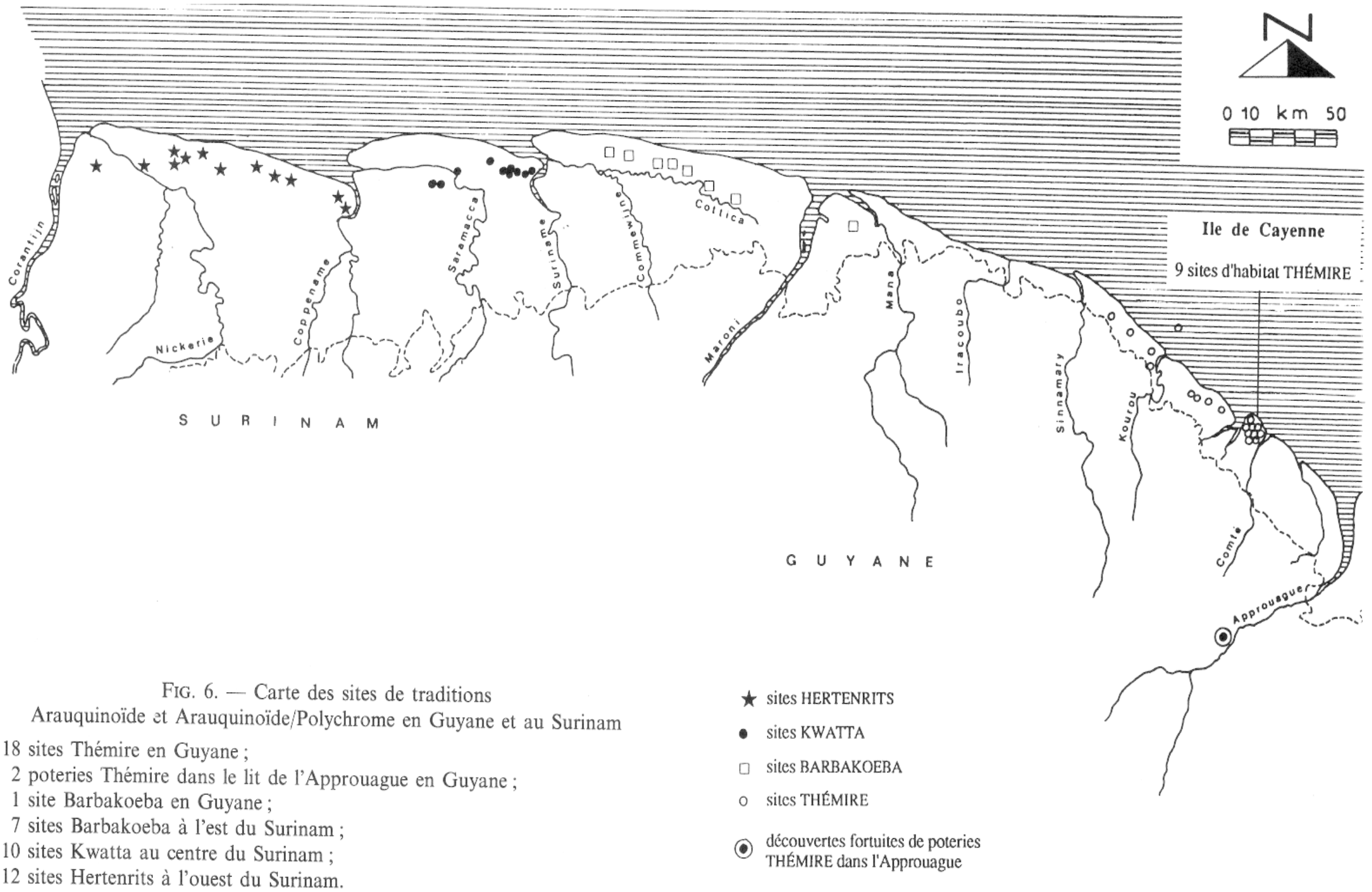


culairement aux cheniers, mesurant 4 à $8 \mathrm{~m}$ de largeur, 1,5 à $2 \mathrm{~m}$ de profondeur et parfois plusieurs kilomètres de longueur.

La céramique, principalement dégraissée avec de la chamotte, présente beaucoup de bols, des jarres, des plaques, et quelques bouteilles. Le décor, présent sur $12 \%$ du matériel, est constitué de cordons ponctués appliqués, de rangées de ponctuations ou d'impressions digitales, d'oreilles géométriques en forme de tête zoomorphe ou anthropomorphe, de colombins apparents.

Le matériel de pierre, retrouvé dans un seul site, est caractérisé par des ciseaux et des lames de hache en pierre polie, dont certaines réutilisées comme percuteurs, des polissoirs, des meules, des percuteurs et quelques fragments d'outils polis. La pierre provenait des groupes spécialisés dans la production de lithiques, du complexe Brownsberg du centre oriental du Surinam (A. Boomert \& S. Kroonenberg, 1977).

\section{2 - Situation du complexe Barbakoeba dans les Guyanes}

La seule datation au ${ }^{14} \mathrm{C}$ du complexe Barbakœba ( $\mathrm{GrN}-7$ 936) donne 1050-1100 de notre ère (A. Versteeg, 1980b). Par comparaisons typologiques, le complexe est daté entre 650 et 1250 de notre ère par Arie Boomert (1993), et entre 1000 environ et au moins 1400 de notre ère par Aad Versteeg (\& F. Bubberman, 1992). Il est vraisemblable que le complexe Barbakoeba du littoral occidental de Guyane date de la même époque que dans l'est du Surinam.

Au Surinam, le complexe Barbakoeba entretenait d'étroites relations avec les complexes littoraux plus occidentaux, Kwatta et Hertenrits, en partie contemporains. La présence de quelques tessons Koriabo dans un site Barbakoeba, et de tessons Barbakoeba dans deux sites littoraux Koriabo indique également l'existence d'échanges entre les deux complexes.

Plusieurs traits céramiques Arauquinoïde caractéristiques, ainsi que la localisation des sites sur chenier et l'utilisation de champs drainés, se retrouvent dans les complexes Barbakoeba et Thémire. Ces concordances indiquent des échanges ou des influences entre les deux complexes et, vraisemblablement, une communauté culturelle. Sur la côte occidentale de Guyane, le complexe Barbakoeba est graduellement remplacé par le complexe Thémire, qui représente quant à lui une fusion des traditions Arauquinoïde et Polychrome.

\section{IV. - LE COMPLEXE THÉMIRE, TRADITIONS ARAUQUINOIDE ET POLYCHROME}

Le complexe culturel Thémire ${ }^{17}$ a été défini sur le littoral central de Guyane, lors de nos recherches. Bien que d'origine Arauquinoïde, il présente de claires influences de la tradition Polychrome. Le territoire Thémire s'étendait de l'Ile de Cayenne jusqu'au Sinnamary au moins, soit approximativement $100 \mathrm{~km}$ de longueur sur 1 à $5 \mathrm{~km}$ de largeur (Figure 6). D'autres sites Thémire seront toutefois peut-être découverts dans le futur plus à l'ouest, jusqu'au fleuve Mana. 


\section{1 - Composantes du complexe ThÉmire en Guyane}

\section{$A-$ Les sites}

Les dix-huit sites connus sont principalement localisés sur des cordons sableux quaternaires, sur des cheniers entre la Karouabo et la rivière de Cayenne et sur des barres prélittorales dans l'Ile de Cayenne. Certains sites de chenier, aujourd'hui localisés au milieu des terres basses, ont pu être côtiers à l'époque de leur occupation, tandis que d'autres, associés à des chemins surélevés se dirigeant vers le rivage, étaient clairement installés en retrait. Quelques sites présentent des localisations différentes, au pied ou au sommet d'une colline, sur une plage. Enfin, la céramique de certains sites des alentours de Petit-Saut, dans le bas Sinnamary (S. Jérémie et al., 1993), nous paraît être des types Thémire ou Barbakoeba. Ces sites intérieurs étaient peut-être des postes Arauquinoïde avancés ou des villages de complexes différents, mais entretenant des relations d'échange avec les populations côtières.

Les sites de cordons sableux présentent plusieurs aires de rejets circonscrites, correspondant peut-être à des maisons communautaires, entourant un espace avec peu de matériel archéologique qui semble avoir été une place centrale. Près de Kourou, trois chemins surélevés (330 à $620 \mathrm{~m}$ de longueur par $5 \mathrm{~m}$ de largeur maximale) ont été aménagés à travers les marais, permettant une communication directe entre des cordons sableux. Il est probable que ces chemins étaient destinés à faciliter la jonction entre des villages et le rivage, vraisemblablement localisé à l'époque au niveau des cheniers septentrionaux.

Par ailleurs, des sites ont pu être spécialisés dans certaines activités, comme par exemple Sainte Agathe qui présentait un nombre particulièrement important de platines.

\section{B - Les champs drainés}

Entre l'Ile de Cayenne et le Sinnamary, de grands ensembles de champs drainés ont été repérés dans les marais, au sud des cheniers présentant les sites (S. Rostain, 1991). Ces champs sont des billons allongés $(30$ à $80 \mathrm{~cm}$ de hauteur, 1 à $3 \mathrm{~m}$ de largeur pour une longueur maximale de $30 \mathrm{~m}$ ) ou des buttes de forme carrée à arrondie ( 20 à $80 \mathrm{~cm}$ de hauteur pour 0,3 à $5 \mathrm{~m}$ de diamètre). Les champs surélevés sont généralement organisés en quadrillage et, souvent, les plus grosses buttes sont situées dans les bas-fonds. Les billons sont orientés dans le sens de la pente en aval des barres prélittorales pour faciliter l'évacuation de l'eau, tandis que les billons plus en amont, par conséquent en milieu moins humide, sont disposés perpendiculairement à la pente pour favoriser au contraire une retenue de l'eau.

Ces champs surélevés avaient pour fonctions la mise en culture de terres noyées, d'une part, et, d'autre part, l'amélioration de la structure du sol par l'aération de la terre avec les terrassements et par le rassemblement des matières organiques superficielles. De nombreux fossés de ceinture au tracé irrégulier $(1$ à $2 \mathrm{~m}$ de largeur) et quelques canaux rectilignes ( $150 \mathrm{~m}$ de longueur sur $2 \mathrm{~m}$ de largeur), 
associés aux champs surélevés, permettaient le drainage de l'eau excédentaire et ont pu servir accessoirement de réservoirs d'eau et de viviers à poissons, ou du moins favoriser leur présence (notamment le coulant et le patagaïe).

Les analyses polliniques réalisées dans divers champs drainés des Guyanes n'ont pas encore révélé la présence de plantes de culture, peut-être à cause de l'important lessivage des sols. Toutefois, cette technique agricole paraît bien adaptée à la culture du manioc amer (Manihot esculenta), du maïs (Zea maïs), de l'igname (Dioscorea sp.) et de la patate douce (Ipomoea batatas). Les premiers textes d'archives et les artefacts liés à la consommation des plantes, retrouvés sur les sites archéologiques, suggèrent que les deux premières cultures ont pu être dominantes.

L'investissement nécessaire à l'édification de champs et de chemins surélevés indique que les sites de cordons sableux ont pu former des centres d'habitat sédentaire. Cette sédentarité était rendue possible par l'abondance de la faune des zones côtières et par des cultures permanentes. La culture sur brûlis nécessite un déplacement régulier des champs et n'est concevable à long terme que pour des groupes relativement restreints. Lorsque les populations augmentent, de nouvelles solutions sont nécessaires. Dans les terres basses inondables, les champs drainés représentent la seule réponse agricole à une augmentation importante du groupe ${ }^{18}$. Ainsi, c'est en période d'accroissement démographique que les Palikur aménagèrent des champs surélevés dans le nord de l'Amapá (P. Grenand, 1981). De telles structures, qui demandent un investissement important en hommes, en temps et en énergie, sédentarisent les groupes autour d'elles. La population Arauquinoïde est estimée entre 300 et 4000 individus par site. Dans l'hypothèse selon laquelle le littoral entre l'Ile de Cayenne et Sinnamary aurait été occupé simultanément par ces agriculteurs et les champs surélevés cultivés de faon continue, la densité humaine aurait pu atteindre de $50 \mathrm{~h} / \mathrm{km}^{2}$ (J. Hurault dans S. Rostain, 1991), ce qui donnerait, en prenant une estimation basse, une population d'approximativement 20000 individus dans cette région.

\section{C-La céramique}

Deux types céramiques au moins caractérisent le complexe Thémire :

Le type Cayenne peint ( 5608 tessons et 23 poteries entières) est caractérisé par un dégraissant de chamotte, pouvant inclure des quantités variables de sable ou de muscovite, une couleur intérieure brun rougeâtre ou grise, de nombreux récipients ouverts petits et moyens et des plaques de grandes dimensions, un décor, présent sur $21 \%$ du matériel, principalement constitué de peinture dichrome rouge et blanche, appliquée uniformément ou en motifs droits et courbes (Figure 7).

Le type Mahury incisé ( 2103 tessons et 4 poteries entières) est caractérisé par un dégraissant de sable quartzeux ou de sable et de mica noir, une cassure principalement de même couleur que la surface ou gris sombre, un décor, présent sur $20 \%$ du matériel, dominé par les droites et les encoches incisées et, plus rarement, des peintures simples monochromes ou dichromes.

Un autre type et une catégorie céramiques, moins bien connus, apparaissent également dans certains sites du complexe Thémire : 

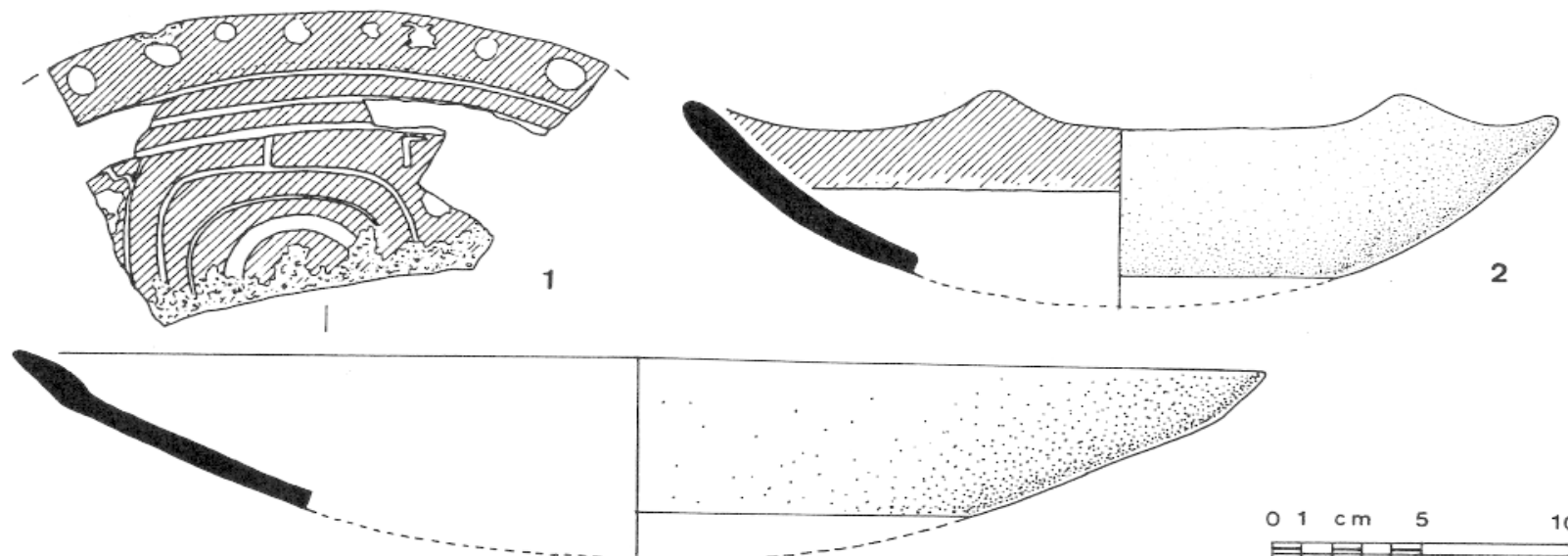

. $1 \mathrm{~cm} 5$

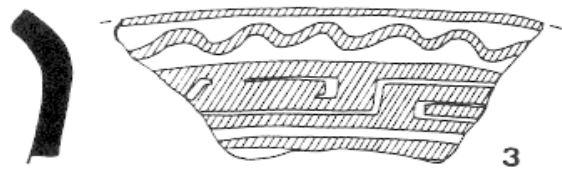

3
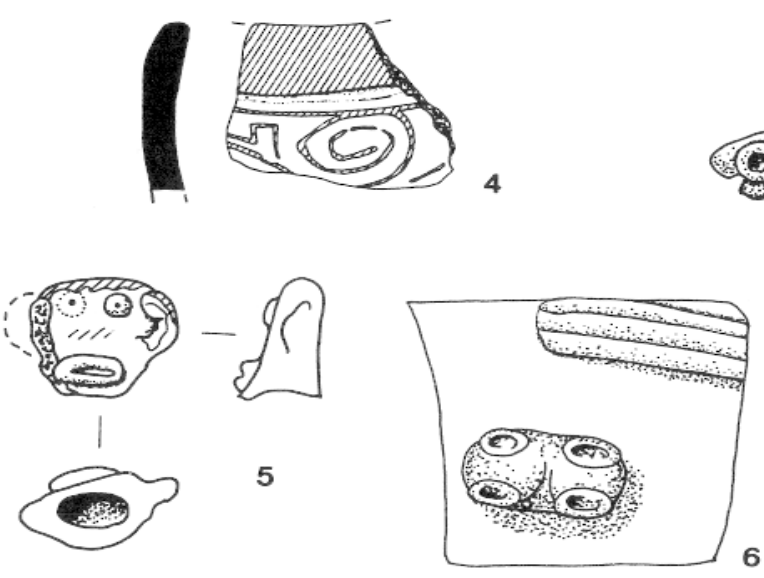

6
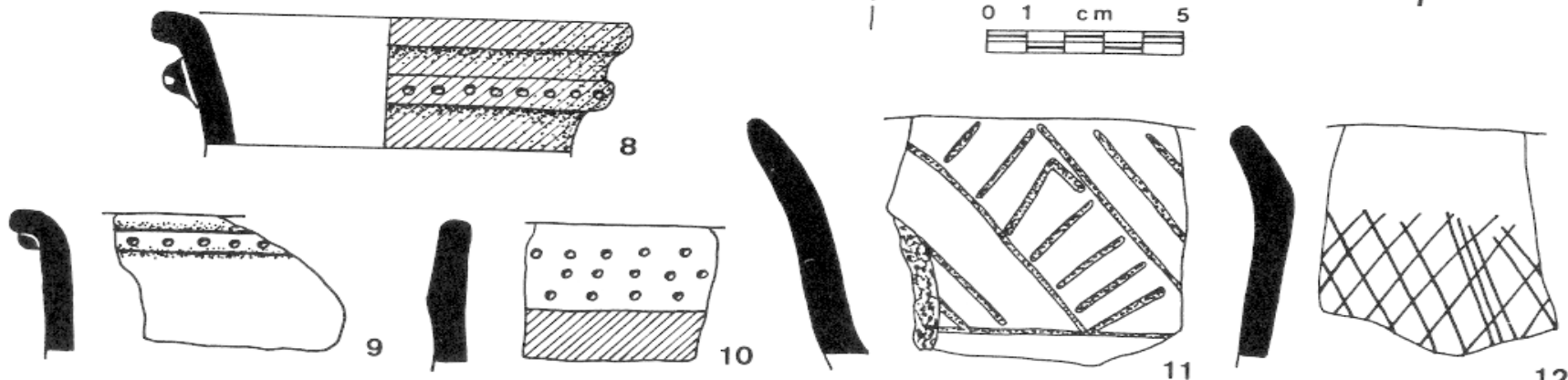

Fig. 7. - Céramiques du complexe Thémire

1) Plat de plan ovale à décor intérieur peint en blanc sur fond rouge, la partie centrale étant très érodée,

Thémire. 2) Plat à bord lobé à décor intérieur peint en rouge et blanc, Thémire. Échelle différente : 3) Bord à décor intérieur peint en blanc sur fond rouge, Sainte Agathe. 4) Bord à décor peint en rouge sur fond blanc, Pascaud. 5) Visage anthropomorphe de lèvre, peint en rouge, Glycérias. 6) Appliqué zoomorphe d'une double tête animale (tortue ?), et d'un triple cordon, Bois Diable. 7) Fragment de grande jarre à modelé zoomorphe (tortue) originellement peinte en rouge, Mini-Circuit Automobile. 8) Bord peint en rouge présentant un cordon appliqué incisé de ponctuations, Bois Diable. 9) Bord avec un cordon appliqué incisé de ponctuations, Pascaud. 10) Bord peint décoré de ponctuations, Pascaud. 11) Bord à incisions diagonales sur l'extérieur, Thémire. 12) Bord à incisions en losanges sur l'extérieur, Anse Legoff. 
La catégorie Montabo rouge (169 tessons) est caractérisé par un dégraissant de sable quartzeux, une pâte d'un rouge caractéristique, un décor de droites incisées.

Le type Melchior kwep (231 tessons et 4 poteries entières) est caractérisé par un dégraissant d'écorces brûlées et broyées, une surface bien lissée, un décor, présent sur $28 \%$ du matériel, uniformément peint en blanc ou rouge, ou faits de droites et d'encoches incisées, de cordons horizontaux dentelés de cupules imprimées avec le doigt. Si ce type, qui apparaît sur le littoral occidental dans le bas Approuague et dans le bas Oyapock, n'est pas encore sûrement attribué à un complexe culturel, on peut toutefois déjà le rattacher partiellement aux complexes Thémire et Koriabo. La définition de ce type céramique demeure provisoire, puisqu'il représente probablement l'amalgame de deux types proches, que le manque de données empêche encore de distinguer.

\section{D - La pierre}

Comme dans la majorité des sites de Guyane, les outils non façonnés dominent (Figure 5). Les lissoirs à céramique sont particulièrement nombreux, confirmant la forte production de poteries qu'indiquait l'abondance des tessons. Les molettes sont également bien représentées, mais les meules sont moins fréquentes. Les autres outils non façonnés apparaissent plus rarement. L'absence de casse-noix pourrait signifier que l'économie des sociétés Thémire était moins dépendante de la cueillette de fruits de palmiers que celle du complexe Aristé. Les outils de pierre polie sont des lames de haches de divers types (Figure 5). Des quartz taillés ont été également retrouvés mais, comme les enclumes/percuteurs, ils sont nettement moins nombreux que dans les sites Aristé. Les râpes étaient apparemment faites de dalles de granite plutôt que de planches à dents incrustées.

Cet outillage, croisé avec les autres données archéologiques, montre que les ressources de ces groupes dépendaient peut-être davantage de l'agriculture que de la cueillette.

\section{2. - Situation du complexe Thémire dans les Guyanes}

\section{A - Les datations}

Les sites des complexes Arauquinoïde du Surinam et de Guyane ont fourni des datations relativement homogènes, entre 600 et 1600 de notre ère environ. Il apparait que les sites occidentaux sont les plus anciens et que les âges rajeunissent vers l'est, ce qui fait penser à un mouvement progressif, mais rapide, venu de l'Orénoque.

Les populations Arauquinoïde, originaires du moyen Orénoque, ont commencé à descendre ce fleuve vers 500 de notre ère, remplaçant progressivement tout du long les anciens complexes Barrancoïde, et entamant les premières reconnaissances vers les Antilles. Dans le delta de l'Orénoque, elles se mêlèrent avec d'autres groupes, comme les Cedeñoïde. 
A partir de cette région, vers $600-700$ de notre ère, de nouveaux mouvements prirent trois directions. Les Cedeñoïde et une partie de la population Arauquinoïde se dirigèrent vers les Llanos occidentaux du Vénézuela, où ils s'installèrent jusqu'à la Conquête. Des groupes mixtes Cedeñoïde-Arauquinoïde-Valloïde, ou un groupe Arauquinoïde acculturé, partirent vers l'île de Trinidad et les Antilles. Enfin, un mouvement Arauquinoïde oriental se diffusa rapidement le long de la côte des Guyanes (A. Boomert, 1993 ; K. Tarble \& A. Zucchi, 1984 ; A. Zucchi, 1991). A partir de 600 de notre ère, des complexes de tradition Arauquinoïde sont détectés sur le littoral occidental surinamien (A. Versteeg, 1985); ils se développèrent dans les Guyanes jusqu'à 1250 de notre ère, certains perdurant même jusque vers 1650 .

Les datations actuelles au ${ }^{14} \mathrm{C}$ du complexe Thémire en Guyane, qui vont de 1400 à 1650 de notre ère, représentent vraisemblablement la fin de ce complexe. Par comparaisons stylistiques avec les données du Surinam, on peut faire remonter le début de ce complexe au moins vers 950 ans notre ère, date la plus ancienne attestée pour les complexes Arauquinoïde orientaux de ce pays (A. Versteeg, 1980b).

\section{B - Le complexe Thémire dans les Guyanes}

Le complexe Thémire représente l'extension littorale la plus orientale, et peut-être la plus récente, de la tradition Arauquinoïde (Figure 6). Il occupe, sur la côte des Guyanes, une place très particulière et originale. En effet, s'il demeure essentiellement rattaché à la tradition Arauquinoïde issue du foyer des moyen et bas Orénoque, il présente parallèlement des caractéristiques de la tradition Polychrome du foyer du bas Amazone. Par l'association de traits de deux grands foyers culturels amazoniens, le complexe Thémire est unique dans l'archéologie de cette région, réalisant la connexion littorale des influences d'Orénoque et d'Amazone.

Le complexe Thémire n'est d'ailleurs pas homogène sur l'ensemble du territoire qu'il occupe. Vers Kourou, et sans doute plus encore vers l'ouest, la céramique montre essentiellement des décors incisés ou appliqués caractéristiques des complexes Arauquinoïde surinamiens : bases spiralées Kwatta, appliqués zoomorphes Barbakoeba, ponctuations et incisions Hertenrits. La peinture rouge est souvent uniformément appliquée, et les motifs dichromes rouges et blancs restent rares. Les similitudes sont plus ténues entre les céramiques des complexes surinamiens et des sites Thémire de l'Ile de Cayenne, où la peinture dichrome est fréquente et la polychromie présente, témoins de l'influence Polychrome dans cette région. Les motifs eux-mêmes, incisés ou peints, sont parfois plus proches du style Aristé que du décor Arauquinoïde (spirales, lignes fines, crans, coins marqués, etc.). En outre, des tessons Aristé, retrouvés dans plusieurs sites de l'Ile de Cayenne, indiquent des échanges dépassant la simple influence. De l'Ile de Cayenne à Kourou, on observe une baisse progressive des décors d'inspiration Aristé, parallèlement à une augmentation des thèmes Arauquinoïde. Les tessons Aristé les plus occidentaux ont été trouvés à Sainte Agathe, près de Macouria.

Ces éléments soulignent le caractère fluctuant des limites d'influence des styles. Si le complexe Thémire représente bien une entité propre, on y perçoit, à l'ouest, des attaches avec le foyer d'Orénoque, et, à l'est, d'autres avec le foyer du bas 
Amazone. Vers 1000-1500 de notre ère, les populations du littoral occidental et central de Guyane, issues du bas Orénoque, développèrent un complexe unique fait d'emprunts aux deux communautés alors les plus puissantes des côtes des Guyanes : Arauquinoïde et Aristé.

\section{V. - LE COMPLEXE KORIABO, TRADITION INCISÉE-PONCTUÉE}

Le complexe Koriabo, originellement défini sur le littoral occidental du Guyana par Clifford Evans et Betty Meggers (1960), fut ensuite reconnu au Vénézuela (J. Cruxent \& I. Rouse, 1959), en Guyane française (F. Bubberman dans D. Grone, 1976), au Surinam (A. Boomert, 1977), sur le littoral oriental du Guyana (A. Boomert, 1978 \& 1979 ; D. Williams, 1978), au Brésil en Amazonas (P. Hilbert, 1982) et dans le bas Xingu (C. Perota, comm. pers., 1990) (Figure 8).

\section{1 - Composantes du COMPleXe Koriabo}

\section{A - Les sites d'habitat}

En Guyane, dix-neuf sites présentent des tessons Koriabo. Dans l'intérieur des terres, neuf d'entre eux sont localisés sur des berges de fleuves. Trois sites sont situés en forêt sur des élévations proches de criques. Les deux sites littoraux sont installés sur des cordons sableux, près d'un cours d'eau. Des céramiques Koriabo intrusives sont parfois retrouvées sur des sites côtiers du complexe Thémire, ainsi qu'un pot unique déposé dans la petite cavité de Ti-Jarre, sur la Montagne Bruyère où tous les autres sites reconnus sont Aristé.

Dans les régions voisines, les sites Koriabo, intérieurs ou côtiers, sont également principalement localisés sur les berges de fleuves ou de petits cours d'eau : 5 sites au Guyana, 22 au Surinam et 1 au Brésil, au nord de l'Amazone. Par ailleurs, des céramiques Koriabo intrusives ont été retrouvées dans 20 sites du Surinam, 8 du Guyana, 1 du delta de l'Orénoque et 1 du bas Xingu (Figure 8).

Cette localisation des sites sur des cours d'eau souvent importants, sur le littoral et, plus tardivement, dans les Iles du Vent, pourrait indiquer que les Koriabo étaient de bons navigateurs, maitrisant bien la fabrication d'embarcations et leur utilisation.

\section{$B$ - La céramique}

À l'origine, la céramique Koriabo de l'ouest du Guyana fut classée en trois types ordinaires, deux types décorés et six catégories (C. Evans \& B. Meggers, 1960). Le décor Koriabo montre une grande homogénéité à travers le large territoire où il est reconnu, les variations d'une région à l'autre intervenant plutôt dans la nature du dégraissant. Si les Koriabo demeuraient partout attachés à leur tradition stylistique, leur technique céramique en revanche s'adaptait aux matières premières locales dont ils disposaient. 


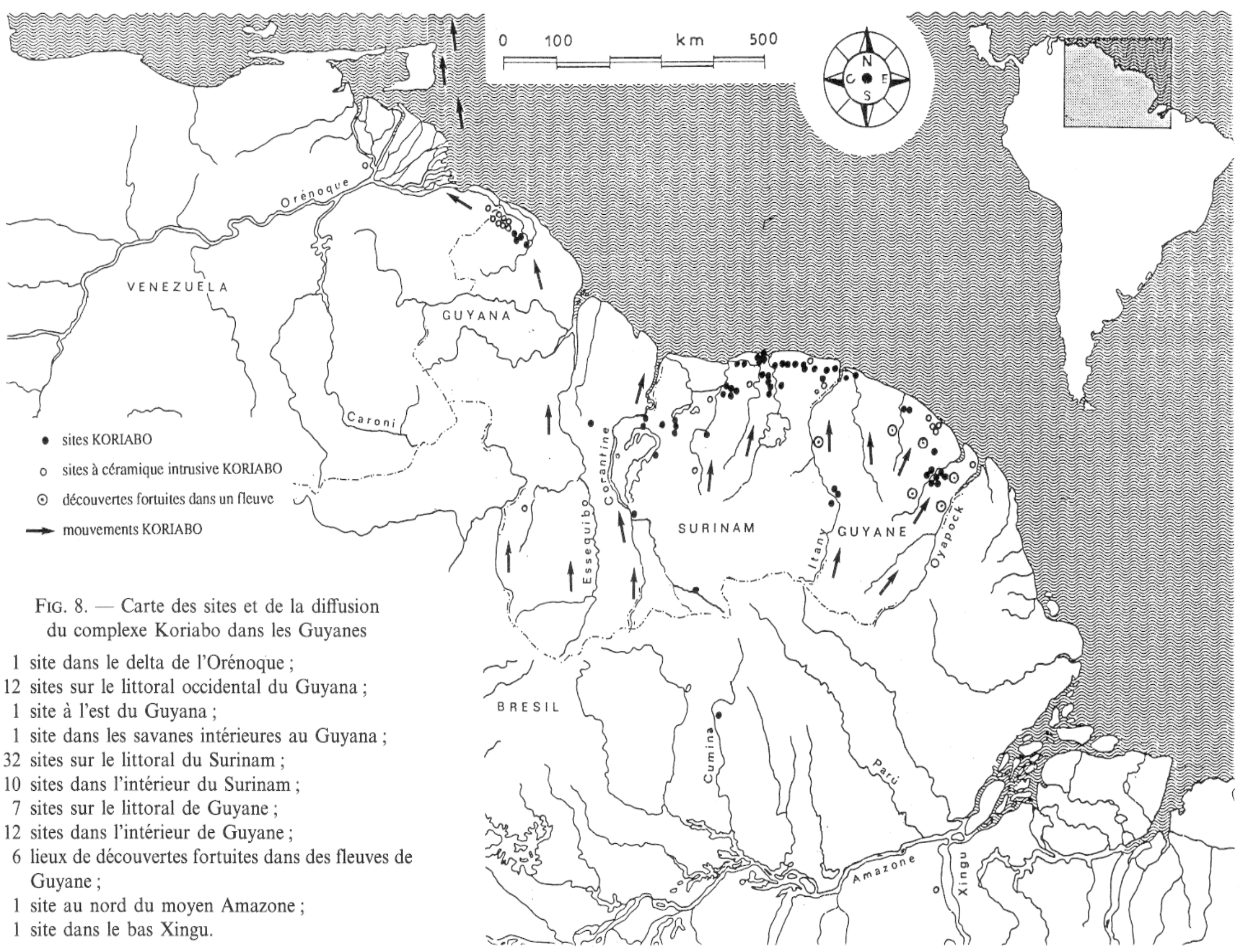


En Guyane, un type céramique au moins caractérise ce complexe. Le type Chaton fantastique (1 661 tessons et 99 poteries entières) est caractérisé par un dégraissant de sable quartzeux fin ou grossier, parfois mélangé à du mica, une pâte de couleur principalement rouge, jaune rougeâtre ou rouge jaunâtre, des formes comprenant un pot très caractéristique à corps globulaire et encolure verticale, un décor rare, présent sur $7 \%$ du matériel, dominé par des incisions fines ou très larges, des cordons et des pastilles annelées appliqués formant parfois des figures zoomorphes ou anthropomorphes (Figure 9).

Le type Melchior kwep ( $c f$. supra « Le complexe Thémire ») apparaît également dans plusieurs sites du complexe Koriabo, auquel il pourrait se rattacher partiellement.

\section{C - Le lithique}

Le matériel lithique est caractérisé par des lames de haches et des ciseaux de pierre polie, des molettes, des meules, des percuteurs, des polissoirs, des lissoirs et des brunissoirs non façonnés, des grattoirs taillés et des éclats de quartz. Des pendentifs zoomorphes de «pierre verte» polie (métabasalte, rhyolite, dolérite, néphrite) ont également été trouvés au Surinam, et il y en avait apparemment aussi en Guyane (Figure 5). Toutefois, peu de caractéristiques remarquables ressortent quant au matériel lithique, somme toute banal dans les sites amazoniens.

En Guyane, le site de Kormontibo, sur le haut Maroni, est particulier car il présentait un nombre relativement important de lames de haches en pierre polie par rapport aux sites des Guyanes, ce qui nous suggère une spécialisation dans la production de lames.

La rareté du matériel lithique découvert dans les sites Koriabo du bas Approuague s'explique par la datation coloniale de ces sites. Plusieurs d'entre eux remontent au XVIII $^{\mathrm{e}}$ siècle, qui connaissait déjà une large diffusion des outils métalliques, précisément dans le bas Approuague où plusieurs habitations coloniales étaient installées. Les Amérindiens de ces sites devaient utiliser ces outils depuis un certain temps et avaient abandonné leurs outils de pierre.

\section{3 - Situation du complexe Koriabo en Amazonie}

\section{A - Les datations}

Le complexe Koriabo fut tout d'abord daté, de façon relative, entre le $\mathrm{xII}^{\mathrm{e}}$ et le $\mathrm{XVI}^{\mathrm{e}}$ siècles de notre ère (C. Evans \& B. Meggers, 1960). Plus récemment, la présence de tessons Koriabo et de matériel européen mélangés dans un site du Guyana fit supposer que le complexe Koriabo avait pu se poursuivre jusqu'au XVIII $^{\mathrm{e}}$ siècle (A. Boomert, 1979).

Des précisions furent apportées par plusieurs datations au ${ }^{14} \mathrm{C}$ obtenues sur des sites surinamiens, mais la position chronologique du complexe Koriabo y est pourtant discutée. Arie Boomert (1986 \& 1993) le date d'entre 950-1000 et 1450 de notre ère. Aad Versteeg, quant à lui (1980b; A. Versteeg \& F. Bubberman, 1992), 

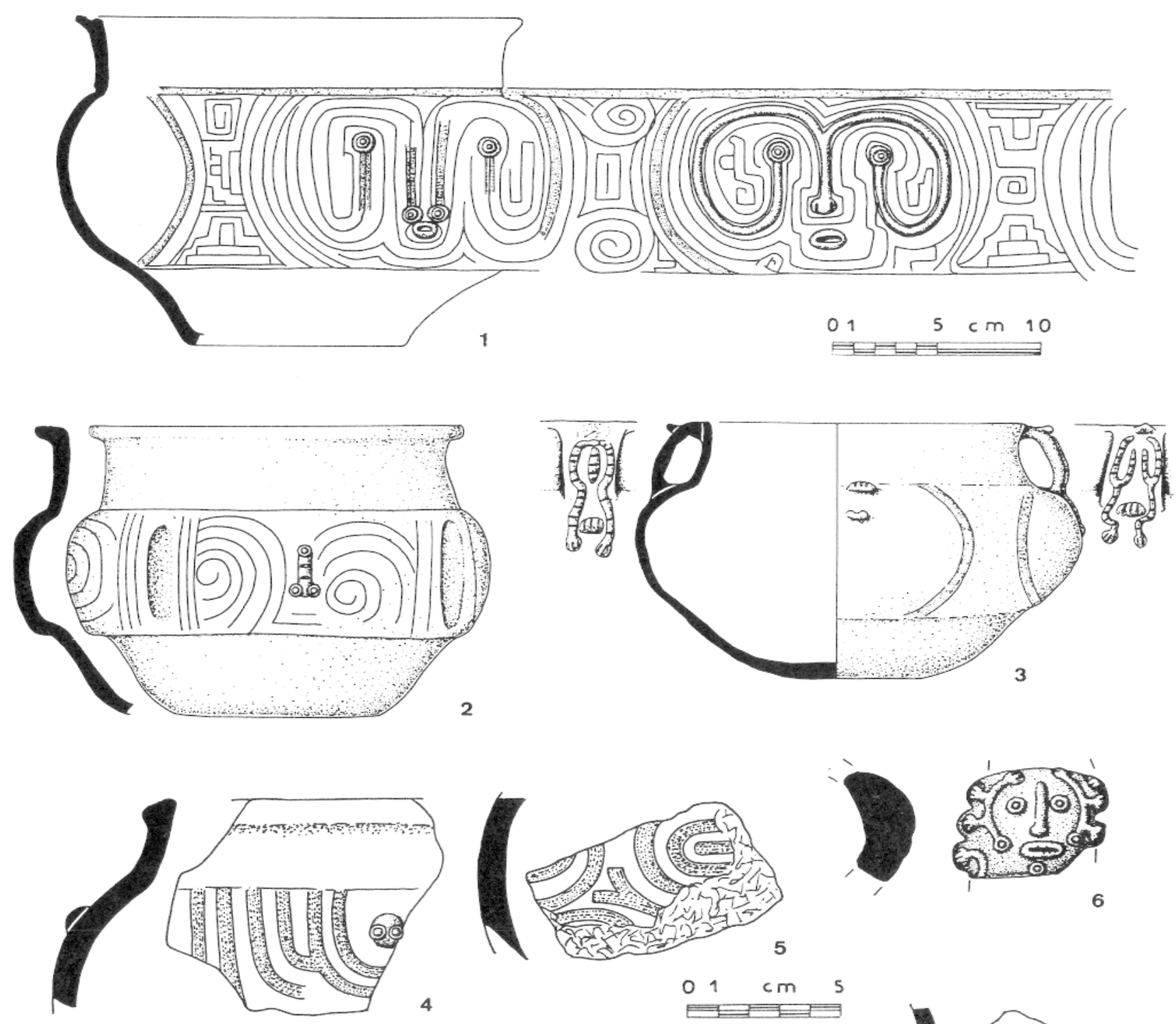

6
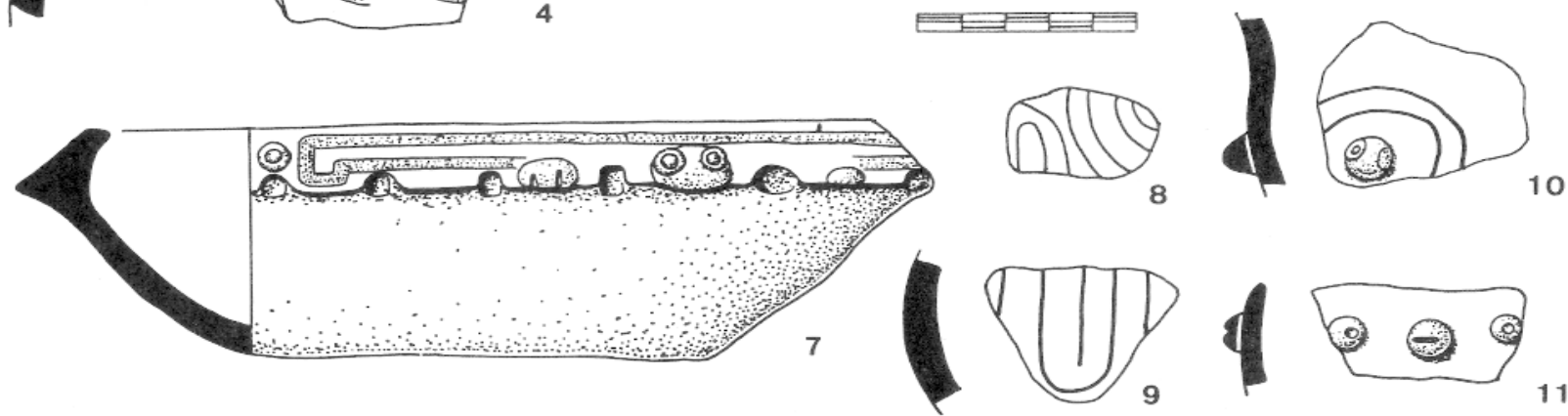

FIG. 9. - Céramiques du complexe Koriabo

1) Pot décoré de quatre visages anthropomorphes sur des gonflements de panse, saut Mapaou. 2) Pot à décor incisé et à modelés appliqués, figurant peut-être des visages anthropomorphes très abstraits, sur quatre gonflements de panse, séparés par des gouttières verticales faites par repousse, crique Bagot. 3) Pot à deux anses décorées de modelés appliquée biomorphes, saut Mapaou. Échelle différente. 4) Bord décoré de larges incisions et de modelés zoomorphes, Mapaou. 5) Tesson de panse décoré de larges incisions, Mapaou. 6) Anse à décor de modelés formant un visage anthropomorphe, P.K. 9,3. 7) Écuelle au bord décoré de modelés zoomorphes appliqués et de larges incisions, saut Mapaou. 8) Tesson de panse décoré d'incisions fines, Mapaou. 9) Tesson de panse décoré d'incisions fines, Mapaou. 10) Tesson de panse décoré d'incisions fines et d'un bouton zoomorphe, Mapaou. 11) Bord décoré de boutons zoomorphes, Piste Météo. 
en se fondant sur les datations au ${ }^{14} \mathrm{C}$, différencie les sites Koriabo intérieurs, datés d'entre 1200 et 1350 environ de notre ère, et les sites Koriabo côtiers plus récents, datés entre 1350 et, au plus tôt, 1600 de notre ère. Par ailleurs, la présence de tessons Koriabo intrusifs et de formes céramiques imitées de ce complexe dans des sites Kwatta et Barbakoeba et, inversement, d'éléments intrusifs et imités de ces derniers dans des sites Koriabo, indique une contemporanéité, au moins partielle, des trois complexes.

En Guyane, la seule datation au ${ }^{14} \mathrm{C}$ du complexe Koriabo, obtenue sur un charbon de bois récolté à Mapaou, dans le bas Approuague, place ce site entre 1520 et 1800 de notre ère (OBDY 728). La carte du bas Approuague de 1764, dressée par Dessingy, indiquant un ancien village akokwa à l'emplacement du site de Mapaou, le calibrage entre 1744 et 1801 (36,2\% de probabilité) est le plus vraisemblable. On peut même resserrer cette fourchette vers sa limite inférieure, c'est-à-dire le milieu du XviII ${ }^{\mathrm{e}}$ siècle. Cet âge, le seul dans les Guyanes qui place de manière sûre le complexe Koriabo à l'époque coloniale, est donc important pour un ajustement de la datation finale de ce complexe, jusqu'alors mal établie, et nous pensons qu'elle représente très probablement l'une des dernières occupations Koriabo de Guyane.

En étudiant l'ensemble des données archéologiques disponibles, nous estimons que les datations du complexe Koriabo de Guyane sont probablement parallèles à celles du Surinam. Les sites de l'intérieur seraient également les plus anciennes occupations, bien que certaines aient pu durer aussi longtemps que dans les sites du littoral. Il est plausible que les sites côtiers Koriabo de Guyane fournissent des âges comparables à ceux des sites littoraux Koriabo surinamiens.

\section{B-Évolution du complexe Koriabo}

Les premières hypothèses faisaient remonter les origines de la culture Koriabo aux complexes Mazagão et Aristé d'Amapá, ces deux derniers étant alors rattachés à la tradition Polychrome (A. Boomert, 1977 ; C. Evans \& B. Meggers, 1960). Nous ne rattachons toutefois pas le complexe Koriabo à la tradition Polychrome mais à la tradition Incisée-Ponctuée qui fut également le foyer de l'ancêtre Aristé-Mazagão (cf. supra).

Il y a quelques années, plusieurs chercheurs supposaient que les populations Koriabo avaient migré depuis l'Amapá le long de la côte vers le nord-ouest, avant de remonter les grands fleuves. Après ce mouvement, qui se serait arrêté sur la rivière Barima au Guyana, elles auraient fui les premiers Européens, pour se réfugier vers l'intérieur des terres (A. Boomert, 1978 ; C. Evans \& B. Meggers, 1960 ; P. Hilbert, 1982; D. Williams, 1985). Leur route aurait alors remonté l'Essequibo, le Rupununi et le Corantyne jusqu'à leurs sources, puis redescendu les rivières Marapi et Cuminá vers l'Amazone (P. Hilbert, 1982).

Les fouilles plus récentes et, surtout, les datations au ${ }^{14} \mathrm{C}$ qui révèlent les sites Koriabo de l'intérieur du Surinam comme plus anciens que les sites littoraux, rendent discutable cette hypothèse de la route littorale de diffusion des populations Koriabo depuis l'embouchure de l'Amazone (A. Versteeg, 1980a). Sur la côte de Guyane, d'autre part, les traces du complexe Koriabo demeurent très ténues dans 
la Montagne Bruyère, l'Ile de Cayenne ou l'embouchure du Mana et deviennent inexistantes sur de larges parties du littoral. En outre, aucun site Koriabo n'est repéré le long de la côte d'Amapá. Ces indices tendent à infirmer le mouvement suggéré plus haut.

Le centre de diffusion de la tradition Incisée-Ponctuée est localisé sur le moyen Amazone, toutefois aucun site Koriabo n'a été découvert dans cette région. Aussi, pour l'instant, il faut admettre que le foyer Koriabo se situerait dans l'intérieur du Plateau des Guyanes plutôt que sur le moyen Amazone. Un mouvement migratoire intérieur direct sud-nord depuis cette région vers le littoral des Guyanes est vraisemblable. Les populations Koriabo se seraient répandues dans les Guyanes en descendant les fleuves qui vont à l'Atlantique (Figure 8). Les sites de berge et les découvertes aquatiques de poteries Koriabo sur ces axes fluviaux attestent le passage de populations de ce complexe, certains sites marquant alors différentes étapes de ce mouvement. Des voies de pénétration fluviale sont actuellement reconnues en Guyane (Oyapock, Approuague, Comté, Sinnamary, Maroni), au Surinam (Tapanahony et Corantyne) et au Guyana (Essequibo et Barima). C'est au cours de ces déplacements que le complexe Koriabo occupa l'intérieur des Guyanes et s'implanta sur quelques sites du littoral entre l'Approuague et l'Orénoque.

Les plus récentes installations Koriabo continentales sont localisées sur la côte des Guyanes et le cours inférieur de leurs fleuves, déjà densément occupés par d'autres communautés. Des sites littoraux des complexes Apostadero, Barbakoeba, Kwatta, Thémire, Mabaruma et Taruma ont livré des céramiques Koriabo, mais c'est avec les complexes Kwatta et Barbakoeba que la cohabitation paraît la plus étroite. Des poteries Kwatta se retrouvent dans plusieurs sites côtiers Koriabo, tandis que des céramiques Koriabo, inversement, sont présentes dans des sites Kwatta. L'apparition fréquente, dans plusieurs sites Barbakoeba et Kwatta, du bol caréné à bord épaissi Koriabo, auquel on suppose un usage cérémoniel, représenterait des échanges inter-communautaires (A. Boomert, 1993). Par ailleurs, on observe fréquemment des imitations de formes céramiques Koriabo par les groupes Barbakoeba et Kwatta au Surinam (A. Boomert, 1993) et par les populations Thémire en Guyane. A l'inverse, aucune imitation de céramiques Arauquinoïde n'apparaît clairement dans la poterie Koriabo. A son arrivée sur la côte des Guyanes, le complexe Koriabo fut donc intégré dans la dynamique inter-tribale et participa au réseau d'échanges local.

Bien que puissante, la culture Koriabo ne se répandit pas uniformément sur le littoral des Guyanes, où elle rencontra des résistances de la part de certaines communautés. Ainsi, les régions côtières dominées par les complexes Hertenrits et Aristé, où aucune céramique Koriabo n'est retrouvée, ne subirent pas cette invasion. Bien plus, cette totale absence de pièces Koriabo indique qu'aucun échange même n'exista entre ces complexes et les Koriabo.

Les groupes Hertenrits, déjà solidement implantés dans la plaine côtière occidentale du Surinam, étaient suffisamment forts pour opposer une bonne résistance (A. Versteeg \& F. Bubberman, 1992). Sur le territoire Aristé, le pot Koriabo déposé dans la cavité de Ti-Jarre constitue la seule exception connue. Il représente une intrusion unique. Aucune autre pièce Koriabo n'ayant été découverte sur l'Oyapock par les orpailleurs en aval de la confluence avec la Camopi, ce 
furent vraisemblablement les habitants Aristé de l'embouchure du fleuve qui stoppèrent l'immigration Koriabo.

L'accroissement démographique que connut le littoral des Guyanes vers 1200-1300 de notre ère chassa apparemment une partie de la population Koriabo vers les Iles du Vent, où elle forma le complexe Cayo.

Le complexe Cayo a été reconnu sur les îles de Saint-Vincent, Tobago, la Dominique, Sainte-Lucie, Grenade et les Grenadines, où il est remplacé vers 1500 par le complexe Fancy (A. Boomert, 1986 ; E. Kirby, 1974). D'étroites similitudes sont observées par Arie Boomert (1986) entre cette céramique et la poterie Koriabo continentale, et la principale différence réside dans la nature du dégraissant, tandis que le décor céramique Cayo est une reprise appauvrie des techniques et des motifs Koriabo. La variété et la complexité moindres des formes et des décors Cayo désignent ce complexe comme une extension insulaire simplifiée du complexe Koriabo. Certaines formes de lames de haches des Petites Antilles également, ainsi que des pétroglyphes, sont similaires à ceux des Guyanes et pourraient de même être associés à cette migration.

À partir du $\mathrm{XVI}^{\mathrm{e}}$ siècle, le complexe Koriabo continental avait beaucoup perdu de ses forces et n'était probablement plus représenté que par de rares villages, notamment au Guyana et en Guyane. Des mouvements vers l'intérieur, contemporains de ceux qui eurent lieu vers les Antilles, sont également possibles.

\section{CONCLUSION : VERS UNE CARTE ETHNO-ARCHÉOLOGIQUE}

\section{A. - Les Aristé}

L'homogénéité matérielle et culturelle du complexe Aristé concorde mal avec la diversité ethnique de l'Amapá à l'époque coloniale. Par ailleurs, l'art actuel des Palikur, groupe dominant en Amapá depuis la Conquête, semble très différent de celui des Aristé. Aussi, avons-nous réalisé une double étude comparative, à partir d'une part, de l'analyse de toutes les pièces archéologiques Aristé, domestiques et cérémonielles, et de l'ensemble de l'art graphique des Palikur, en interrogeant d'autre part des potières palikur (S. Rostain, 1990) auxquelles furent soumis des dessins de formes et de décors céramiques Aristé.

Peu de convergences apparaissent de prime abord entre les styles Aristé et palikur, où seuls quelques éléments décoratifs simples sont comparables, tandis que les formes diffèrent presque toutes, ainsi que les dégraissants. Si les potières palikur ne reconnaissent pas les décors courbes, en volutes et en lignes élaborées de l'Aristé Récent, elles sont en revanche plus enclines à attribuer à leur groupe certains motifs linéaires, crénelés ou triangulaires des traditions Incisée-Ponctuée (complexes Mazagão et Aristé Ancien) ou Arauquinoïde.

À travers l'étude stylistique de la céramique de certaines nécropoles, en revanche, nous avons pu retracer une évolution probable de la céramique Aristé vers celle des Palikur actuels. Il semble que les poteries de nécropoles d'époque historique témoignent de l'émergence de styles exogènes au sein du sous-complexe 
Aristé Récent. Les formes et les décors céramiques s'éloignent progressivement des caractéristiques de la poterie Aristé Récent, pour se rapprocher de ceux des complexes Mazagão et Arauquinoïde. La fusion des complexes Aristé, Mazagão et Arauquinoïde constituerait donc la genèse de l'art céramique des Palikur actuels, avec un style hybride évoluant progressivement pour aboutir à un type céramique original. Au début du $\mathrm{xx}^{\mathrm{e}}$ siècle, la poterie palikur possédait déjà les caractéristiques (C. Nimuendaju, 1926) que nous observons aujourd'hui, et aucune migration amérindienne ni influence extérieure notable n'a, depuis lors, altéré ce style.

Cette évolution dans la céramique s'accompagna de l'abandon progressif des anciennes coutumes funéraires par crémation, qui semblent dominer dans le sous-complexe Aristé Récent, au profit de la sépulture secondaire après décharnement, apparemment déjà caractéristique du complexe Mazagão. Ce schéma a l'avantage de s'accorder avec l'histoire des origines des Palikur, qui nous est transmise par leur tradition orale (P. \& F. Grenand, 1987) et par les archives. La crémation était encore contemporaine de la sépulture secondaire chez les Palikur à la fin $\mathrm{du} \mathrm{XVII}^{\mathrm{e}}$ siècle. Puis, au $\mathrm{XVIII}^{\mathrm{e}}$ siècle, seule la sépulture secondaire était signalée, avant d'être définitivement remplacée, vers 1960 et sous la pression des religieux, Jésuites et missionnaires protestants, par l'enterrement chrétien.

Des réfugiés arrivèrent sur la côte septentrionale d'Amapá, principalement des Antilles et du bas Orénoque au $\mathbf{X V I I}^{\mathrm{e}}$ siècle, et de l'embouchure de l'Amazone, au $\mathrm{XVII}^{\mathrm{e}}$ et au XVIII ${ }^{\mathrm{e}}$ siècle, fuyant les guerres territoriales européennes et les chasses aux esclaves qui sévissaient dans ces régions.

Des ethnies, dont certaines probablement rattachées à la tradition Arauquinoïde qui dominait alors dans le bas Orénoque, migrèrent en apportant des traits céramiques Arauquinoïde, qui apparaissent dans la poterie palikur actuelle. Ce sont peut-être également ces communautés Arauquinoïde qui enseignèrent aux Palikur la technique des champs surélevés, qu'ils employèrent au $\mathrm{XvII}^{\mathrm{e}}$ siècle.

L'immigration vers la Guyane de populations de l'embouchure de l'Amazone est signalée dans les archives, et par la tradition orale palikur. Celle-ci dit que deux des clans palikur qui arrivèrent du sud de l'Amapá introduisirent la langue palikur actuelle, la râpe à dents de métal et la culture du manioc (P. \& F. Grenand, 1987). Certains de ces clans habitaient auparavant sur la rivière Maraca, foyer du complexe Mazagão, et nous pensons que ces ethnies méridionales appartenaient à ce complexe. On peut supposer qu'elles donnèrent également certains traits de leur style céramique aux Palikur du nord de l'Amapá. L'origine commune de ces complexes septentrionaux et méridionaux, ainsi que les échanges cérémoniels qu'ils entretinrent en dépit d'évolutions relativement indépendantes, facilitèrent sans doute la fusion, à l'époque de la Conquête, des populations Aristé et Mazagão dans le nord de l'Amapá ; ils formèrent, en se conjuguant aux styles Arauquinoïde, l'art céramique et la culture palikur actuels.

Si les Palikur semblent aujourd'hui être les principaux héritiers indirects des complexes archéologiques Aristé et Mazagão, d'autres nations ont participé autrefois à la dynamique de ces ensembles culturels. A partir des archives et de la tradition orale palikur, Pierre et Françoise Grenand (1987) distinguent, sur la côte d'Amapá et depuis l'époque de la Conquête au moins, 18 clans palikur et 23 autres ethnies. Ces auteurs estiment qu'à la période coloniale, une compétition entre 
nations induisit "un mouvement de relative unification régionale centrée sur les Palikur » (1987, page 16). Cette unification devenait particulièrement effective en temps de guerre.

Cette confédération historique pan-tribale semble être un héritage direct de la préhistoire, durant laquelle elle était matérialisée par le complexe Aristé. L'unité matérielle, la délimitation géographique précise et l'extrême rareté de pièces intrusives, sont les indices de la grande homogénéité de ce complexe. Les diverses ethnies partageaient une culture matérielle commune et vivaient dans un système d'échange local. Le nombre restreint d'urnes dans chaque nécropole Aristé laisse supposer que ce mode de sépulture était réservé aux personnages importants, chefs de clan ou d'ethnie. En outre, le nombre relativement important de cimetières, contenant chacun peu de pièces, et la variabilité du décor des urnes funéraires de l'un à l'autre, sont peut-être des indices de divisions ethniques ou claniques. Ces particularismes seraient la manifestation de l'identité propre à chaque unité au sein d'un ensemble culturel homogène ${ }^{19}$.

\section{B. - Les Arauquinoïde}

Si la diversité ethnique ancienne d'Amapá est bien rendue par les archives, le reste de la côte des Guyanes apparaît relativement plus uniforme, avec la domination des groupes aruak à l'époque de la Conquête. L'archéologie montre par ailleurs, de 500 à 1650 de notre ère environ, le développement d'un ensemble culturel homogène Arauquinoïde qui s'étend, sur la côte, du delta de l'Orénoque à l'Ile de Cayenne, avec des variations locales et progressives qui justifient la définition de différents complexes. Les comparaisons déjà réalisées entre des familles linguistiques et des traditions archéologiques se fondent principalement sur la similitude des aires d'occupation et de diffusion, indiquant que la famille linguistique aruak-maipuran serait porteuse des traditions Barrancoïde puis Arauquinoïde (D. Lathrap, 1970 ; K. Tarble \& A. Zucchi, 1984 ; A. Zucchi, 1988 \& 1991).

Sur la côte centrale de Guyane, des influences stylistiques Aristé sont parfois repérées dans la céramique Thémire ${ }^{20}$. Ces influences, ainsi que la présence de pièces intrusives Aristé dans l'Approuague et dans l'Ile de Cayenne, proviennent apparemment de commerçants ou de déplacements de petits groupes Aristé, tels ceux signalés dans les archives à l'époque historique.

Plusieurs indices archéologiques suggèrent que les Arauquinoïde étaient organisés en chefferies, avec une centralisation du pouvoir et une division du travail fondées sur la spécialisation et la complémentarité.

\section{C. - Les Koriabo}

L'aire des anciens mouvements et occupations karib correspond relativement bien à la carte des sites Koriabo ; aussi des parallèles entre les deux ont-ils été tentés (A. Boomert, 1986). 
Divers groupes de la famille linguistique karib sont répartis dans l'intérieur du Plateau des Guyanes et la carte de leurs migrations anciennes, dressée principalement à partir de données linguistiques, concorde approximativement aux mouvements supposés des populations Koriabo. Sur la côte des Guyanes, les principaux groupes karib historiques étaient les Karinya à l'ouest, et les Galibi en Guyane.

Par ailleurs, diverses autres ethnies utilisèrent la céramique Koriabo en Guyane. Les fouilles que nous avons réalisées dans des sites précisément localisés sur une carte du $\mathrm{XVIII}^{\mathrm{e}}$ siècle ont montré que les Norak (groupe linguistique tupi) et les Akokwa (karib ?), qui vivaient apparemment en bons termes avec les Galibi, occupaient des sites du complexe Koriabo dans le bas Approuague. Comme pour les Aristé, le complexe Koriabo était donc représenté par des ethnies de groupes linguistiques divers. En outre, il faut noter que les Norak s'intégrèrent tardivement à ce complexe, puisqu'ils n'arrivèrent qu'à l'époque historique sur l'Oyapock et l'Approuague (P. Grenand, 1971 \& 1982 ; J. Hurault, 1972). Il furent, par ailleurs, parmi les derniers à utiliser de la poterie Koriabo, jusqu'à leur propre extinction, au XVII ${ }^{\mathrm{e}}$ siècle. Cela montre qu'un complexe culturel pouvait se maintenir dans une région alors que les populations changeaient.

Ces éléments nous incitent à penser que le complexe Koriabo était originellement représenté par au moins plusieurs ethnies de famille linguistique karib. Sur la côte des Guyanes, les Koriabo rencontrèrent les populations Arauquinoïde, avec lesquelles ils fusionnèrent, pour donner naissance, après avoir reçu quelques influences limitées des styles Aristé, à de nouveaux styles céramiques hybrides. En Guyane, la céramique galibi actuelle serait l'héritage de ces multiples influences.

Plus qu'un simple complexe culturel étendu, le complexe Koriabo représente un très vaste mouvement migratoire, depuis le Plateau des Guyanes jusqu'aux Antilles, de populations qui, en moins de 200 ans, se fondirent avec la plupart des groupes indigènes des régions traversées.

\section{D. - Conclusion}

Le littoral de Guyane occupe une place unique dans les développements culturels préhistoriques tardifs du Plateau des Guyanes. L'Ile de Cayenne marque une frontière nette autant qu'un lieu de rencontre de diverses influences. Au sud-est, la confédération Aristé est reliée au foyer du bas Amazone, tandis que la côte centrale et occidentale est dominée par les chefferies Arauquinoïde, issues du foyer du bas Orénoque, et que les groupes Koriabo, provenant de l'intérieur du Plateau des Guyanes, occupent les axes fluviaux et tentent de s'implanter sur les espaces peu densément habités du littoral. A la jonction de ces trois mouvements, l'Ile de Cayenne fut au centre de mélanges culturels et ethniques.

D'importants bouleversements culturels ont touché le littoral des Guyanes vers 1100-1300 de notre ère avec, notamment, l'arrivée et le départ de diverses populations. La carte culturelle de la côte des Guyanes se trouva alors modifiée peu avant la Conquête, et les premiers explorateurs européens y rencontrèrent des 
sociétés récemment restructurées. Ce contact entre deux mondes allait encore, à son tour, changer ce nouveau visage*.

* Manuscrit reçu en février 1994, accepté pour publication en juin 1994.

\section{NOTES}

1. De 1988 à 1991, au département d'archéologie du centre ORSTOM đe Cayenne, nous avons mené le programme de recherche sur l'occupation amérindienne ancienne du littoral de Guyane, objet d'une thèse de Doctorat, soutenue en février 1994 à l'université de Paris-I/Panthéon-Sorbonne. Le texte présenté ici reprend quelques-unes des principales conclusions de cette thèse.

2. Comme aucune typologie céramique n'avait été définie en Guyane, et comme il n'existe pas de méthodologie encore bien adaptée au matériel amazonien, nous avons adopté une classification préliminaire simplifiée ; il sera nécessaire dans le futur de distinguer de nouveaux types et de subdiviser certains de ceux qui existent en plusieurs variétés.

3. Dès les premiers contacts, les Européens échangèrent des perles de verre (la pacotille) et des outils métalliques, principalement, contre de la nourriture et des productions artisanales amérindiennes.

4. Nous n'accordons pas le nom des traditions et des complexes, qui conservent également leurs majuscules.

5. Aucune fouille archéologique n'a été menée dans l'intérieur de l'Amapá, et il est possible que des sites Aristé soient découverts en forêt dans le futur. Il faut toutefois noter que l'influence des complexes littoraux des Guyanes se limite généralement à la plaine côtière.

6. Les abris-sous-roche des collines de Ouanary sont formés naturellement par érosion et effondrement dans des affleurements de la carapace latéritique.

7. D'après le code Munsell des couleurs.

8. Ce dégraissant est appelé $k w e p$ par les Palikur, qui l'utilisent encore aujourd'hui.

9. L'outillage taillé est essentiellement fait sur du quartz local, le quartzite n'apparaissant que très ponctuellement.

10. La datation de 164-39 avant notre ère (OBDY 653) du site de Carbet Mitan se distingue nettement des autres âges obtenus pour le sous-complexe Aristé Ancien ; aussi, en l'absence d'autres datations aussi anciennes, préférons-nous pour l'instant ne pas la retenir.

11. Les faïences sont trop récentes pour avoir été échangées par les Hollandais qui, dès 1598, mirent en place d'intenses relations commerciales avec les populations côtières des Guyanes, puis installèrent, jusqu'à 1647 seulement, plusieurs petites colonies au nord de l'Amapá et sur le bas Oyapock (B. Meggers \& C. Evans, 1957).

12. Ce type nous a servi à définir le sous-complexe Aristé Moyen, celui-ci ne séparant toutefois pas de façon chronologique les sous-complexes Aristé Ancien et Aristé Récent, qui se chevauchent, mais marquant l'intrusion d'une nouvelle population dans la séquence.

13. Les styles céramiques de ces deux complexes sont très proches. Le premier remonterait à environ 200 de notre ère (M. Simões \& A. Machado, 1987). Le second est daté relativement de 1000 à 1500 de notre ère (P. Hilbert, 1968), mais les arguments utilisés pour ce placement chronologique sont peu convaincants, et diverses autres données tendent à indiquer que ce complexe pourrait être plus ancien.

14. "Un chenier est un type de plage sableuse (ou très riche en débris de coquillages) formée sur un substratum de sédiments fins (argiles, limons) dans une côte caractérisée par des phénomènes d'accrétion et de recul " (M.-T. Prost, 1990, page 19).

15. La terra preta (terre noire), fertile, est le résultat de la transformation physico-chimique de rejets anthropiques anciens.

16. Le site de Route de Mana P.K. 26,5 présente de la céramique Koriabo, mais le matériel céramique n'est pas encore complètement étudié. Si ce site s'avère réellement Koriabo, il n'est pas impossible que des pièces Barbakoeba échangées y soient reconnues, comme c'est le cas dans plusieurs sites Koriabo du Surinam. A l'inverse, ce site est peut-être Barbakoeba avec des pièces Koriabo intrusives.

17. Le terme de Thémire a été donné par référence au site du même nom de l'Ile de Cayenne. 
18. L'agriculture sur brûlis devait se poursuivre parallèlement ailleurs, notamment sur les collines de l'Ile de Cayenne.

19. La division clanique des Palikur ainsi que l'existence, encore récente, de cimetières différenciés, sont probablement les derniers reflets de ce que fut la confédération pan-tribale Aristé.

20. À l'inverse, aucun trait Thémire ne semble apparaître dans le complexe Aristé et, avant les migrations provoquées par la Conquête, il semble que l'influence des Arauquinoïde ne s'étendit pas au-delà de l'Ile de Cayenne.

\section{RÉFÉRENCES CITÉES}

BOOMERT, Arie, 1977. - «Prehistorie ». Encyclopedie van Suriname. Amsterdam, Elsevier, Brussel. Traduction en anglais par A. Boomert, 506-517.

—, 1978. - « Report on ceramic samples from Itabru, Berbice River ». Journal of the Walter Roth Museum of Archaeology and Anthropology. Vol. I, $\mathrm{n}^{\circ}$ 1, Georgetown, 36-39.

-, 1979. - " An analysis of the ceramic finds from Itabru, Berbice river ". Journal of the Walter Roth Museum of Archaeology and Anthropology. Vol. II, $\mathrm{n}^{\circ}$ 1, Georgetown, 7885.

-, 1980. - "The Sipaliwini Archaeological complex of Surinam : a summary ». Nieuve West-Indische Gids. $\mathrm{N}^{\circ}$ 2, Stichting Nieuwe West-Indische Gids, Utrecht, 94-107.

-, 1986. - « The Cayo complex of $S^{t}$ Vincent : ethnohistorical and archaeological aspects of the island carib problem ». Antropológica, 66, Fundación La Salle, Instituto Caribe de Antropología y Sociología, 3-68.

- 1993. - "The Barbakoeba archaeological complex of northeast Suriname». OSO : Tijdschrift voor Surinaamse Taalkunde, Letterkunde, Cultuur en Geschiedenis. 12, 2, 198-222.

BoOmert, Arie \& KroOnenberg, Salomon B., 1977. - « Manufacture and trade of stone artifacts in Prehistoric Surinam ". Ex Horreo IPP 1951-1976. Cingula 4. B.L. Van Beek, R.W. Brandt and W. Grœmman-Van Waateringe éds., Amsterdam, 9-46.

CORNETTE, Alain, 1985. - Site archéologique de Crique Jacques (Mana, Guyane française). A.G.A.E., Cayenne, Ms, 10 pages +18 figures.

CruXent, José M. \& Rouse, Irving, 1959. - An archaeological chronology of Venezuela. 2 vols., Pan American Union, Social Science Monograph $\mathrm{n}^{\circ}$ 6, Washington.

Evans, Clifford \& Meggers, Betty J., 1960. - Archeological investigations in British Guiana. Smithsonian Institution, Bureau of American Ethnology, bulletin 177, Washington.

Grenand, Pierre, 1971. - Les relations intertribales en haute Guyane du XVIT siècle à nos jours. Maîtrise spécialisée d'ethnologie.

-, 1981. - «Agriculture sur brûlis et changements culturels : le cas des Indiens Wayãpi et Palikur de Guyane ». Journal d'Agriculture Traditionnelle et de Botanique Appliquée. XXVIII, 1, 23-31.

-, 1982. - Ainsi parlaient nos ancêtres. Essai d'ethnohistoire "Wayãpi ». Travaux et documents de l'ORSTOM, $\mathrm{n}^{\circ} 178$, ORSTOM éd., Paris.

Grenand, Pierre \& Grenand, Françoise, 1987. - « La côte d'Amapá, de la bouche de l'Amazonie à la baie d'Oyapock, à travers la tradition orale Palikur ». Boletim do Museu Paraense Emilio Galdi, Série Antropologia, 3, 1, Belém, 1-77. 
Grane, Denis, 1976. - « Note sur le site de Kormontibo ». Compte rendu du VI Congrès International d'Étude des Civilisations Précolombiennes des Petites Antilles. Centre Universitaire Antilles Guyane. Pointe-à-Pitre, Guadeloupe, Département d'Histoire et d'Archéologie, 158-164.

Hilbert, Peter, 1968. - Archäologische Untersuchungen am mittleren Amazonas. Marburger Studien zur Völkerkunde 1, Berlin, Dietrich Reimer Verlag.

-, 1982. - " Pottery from the Cumina river, Brazil, and its Affiliations with the Koriabo Phase of Guyana ». Journal of the Walter Roth Museum of Archaeology and Anthropology. V, 2, Georgetown, 74-81.

Hurault, Jean, 1972. - Français et Indiens en Guyane (1604-1972). Coll ${ }^{\circ} 10 / 18, \mathrm{n}^{\circ} 690$ Paris, U.G.E..

JÉRÉMIE, Sylvie et alii, 1993. - " Archéologie de sauvetage en Guyane française : le chantier de Petit-Saut, bilan en juin 1993 ». Journal de la Société des Américanistes, LXXIX, Paris, 211-224.

KIRBY, I. A. Earle, 1974. - «The Cayo pottery of $S^{t}$ Vincent. - a pre-Calivigny series ». Coinpte rendu du $V^{e}$ Congrès International d'Etude des Civilisations Précolombiennes des Petites Antilles. Antigua, The Antigua Archaeological Society, 61-64.

LathraP, Donald W., 1970. - The upper Amazon. London, Thames \& Hudson, Glyn Daniel general editor.

Meggers, Betty J. \& Evans, Clifford, 1957. - Archeological investigations at the mouth of the Amazon. Smithsonian Intitution, Bureau of American Ethnology, bulletin 167, Washington.

Nimuendaju, Curt, 1926. - « Die Palikur Indianer und ihre Nachbarn » (les Indiens Palikur et leurs voisins). Kongl. Vet. Vitt. Hand. 31 (2). Göteborgs. Traduction de Claudie Jousse, 1971.

Nordenskiold, Erland, 1930. — «'archéologie du Bassin de l'Amazone ». Ars Americana. $\mathrm{N}^{\circ}$ 1, Paris.

Nowacki-Breczewski, Philippe \& Puaux, Olivier, 1992. - Sauvetage archéologique de Petit-Saut. Rapport général janvier 1991-mai 1992. Association pour les fouilles archéologiques nationales, Service régional d'archéologie (D.R.A.C. Guyane), Conseil régional de la Guyane, Électricité de France, Cayenne, Ms, 84 pages + planches.

Petitjean Roget, Hugues, 1980. - « La fouille de la grotte Trou Biche : contribution à l'étude du site archéologique de la montagne Bruyère dans le bas-Oyapock ». Équinoxe, 13, Revue Guyanaise d'Histoire et de Géographie, C.E.G.E.R., Cayenne, 41-46.

-, 1993. - Fouille de sauvetage urgent. Site $n^{\circ}$ 97-1-12-314-16, Trou Delft : un site funéraire post-colombien sur l'Oyapock en Guyane française. Communication au $\mathrm{XV}^{\mathrm{e}}$ Congrès de l'Association Internationale d'Archéologie des Caraïbes, San Juan de Porto-Rico, multigr. 9 pages.

Prost, Marie-Thérèse, 1990. - «Modifications des rivages et conditions actuelles de formation des cheniers sur les côtes de Cayenne". Symposium international PICG 274/ORSTOM, Guide de l'excursion A : Presqu'île de Cayenne. Géomorphologie, Cayenne, 16-30.

Prous, André, 1986. - "L'archéologie au Brésil, 300 siècles d'occupation humaine ». L'Anthropologie. 90, 2, Paris, 257-306.

Roosevelt Anna C., Housley R., Imazio M., Maranca S. et Johnson R., 1991 - « Eight millenium pottery from a prehistoric shell midden in the brazilian Amazon ». Science, 13 décembre, 254, $1621-1624$. 
Rostain, Stéphen. - 1990. - L'art de la céramique amérindienne en Guyane. Document pédagogique, série Art \& archéologie, $\mathrm{n}^{\circ} 1$, Inspection académique de la Guyane/Ministère de la Culture, Cayenne.

—, 1991. - Les champs surélevés amérindiens de la Guyane. Coll. « la Nature et l'Homme ", centre ORSTOM de Cayenne.

-, 1994. - L'occupation amérindienne ancienne du littoral de Guyane. Thèse de Doctorat, Université de Paris-I/Panthéon-Sorbonne, U.F.R. d'Art et d'Archéologie, Centre de Recherche en Archéologie Précolombienne, deux tomes, multigr. 718 pages +216 figures +31 photographies.

SCHсEPF, Daniel, 1972. - « Historique et situation actuelle des Indiens Wayana-Aparai du Brésil ». Bulletin du Musée d'Ethnographie de Genève. 15, 33-64.

Simces, Mario F., 1981. — «Coletores-pescadores ceramistas do littoral do Salgado (Para)». Boletim do Museu Paraense Emilio Goldi, Nova série, Antropologia, 78, Belém, 1-32.

-, 1983. - «A pré-historia da Bacia Amazônica (uma tentativa de reconstituição)». Cultura indigena, Semana do Indio, exposição temporaria, Museu Paraense Emilio Gœldi, Belém, 5-12.

Simces, Mario F. \& Machado, Ana Lucia 1987. - «Pesquisas arqueológicas no lago de Silves (Amazonas) ». Revista de Arqueologia, 4, 1, MPEG/CNPq, Belém, 79-82.

TARBle, Kay \& ZuCCHI, Alberta, 1984. - « Nuevos datos sobre la arqueología tardía del Orinoco : la serie valloide », Acta Científica Venezolana, 35, 434-445.

Turenne, Jean-François, 1974. - « Le gisement de Pointe Gravier, Guyane francaise ». Compte rendu du $V^{e}$ Congrès International d'Etudes des Civilisations Précolombiennes des Petites Antilles. Antigua, The Antigua Archaeological Society, 28-34.

Versteeg, Aad H., 1978. - «A distinctive kind of pottery in western Suriname». Mededelingen Surinaams Museum, 23 et 24, Paramaribo, 16-27.

-, 1980a. - "Archaeological investigations at Kwamalasamœtœ, south Suriname". Mededelingen Surinaams Museum, 30, Paramaribo, 22-43.

-, 1980b. - « C-14 datings from archaeological sites in Suriname». Mededelingen Surinaams Museum, 32, Paramaribo, 38-56.

-, 1985. - The Prehistory of the Young Coastal Plain of West Suriname. Berichten van de Rijksdienst voor het Oudheidkundig Bodemonderzœk, Jaargang 35, 653-750.

Versteeg, Aad H. \& Bubberman, Frans C., 1992. - Suriname before Columbus. Stichting Surinaams Museum, Mededelingen, $\mathrm{n}^{\circ} 49 \mathrm{a}$, Paramaribo. 64 pages.

WILliams, Denis, 1978. - «Tests pits at Itabru, Berbice River ». Journal of the Walter Roth Museum of Archaeology and Anthropology, I, 1, Georgetown, 34-35.

-, 1985. - Ancient Guyana. Department of Culture, Georgetown. 94 pages.

ZucCHI, Alberta R., 1988. - « Linguística, Etnografia, Arqueología y cambios climáticos : la dispersión de los Arawaco en el Noroeste Amazónico ". Communication au Congrès International des Américanistes, Série « Archéologie et milieu ambiant ", $46^{\circ}$, Amsterdam, 34 pages +12 pages de figures.

—, 1991. - « La serie meillacoide y sus relaciones con la cuenca del Orinoco ». Comptes rendus du XI Congrès de l'Association Internationale d'Archéologie des Caraïhes. San Juan de Puerto Rico, A. Tekakis, I. Vargas Arena et M. Sanoja Obediente éds. Fundación Arqueológica, Antropológica e Histórica de Puerto Rico/Universidad de Puerto Rico/ United States Forest Service, 272-286. 


\section{ANNEXE 1 : LISTE DES SITES DE GUYANE CLASSES DANS DES COMPLEXES CULTURELS}

\section{ARISTE}

Plein air :

Fort Saint-Louis

Saut Maripa

Pointe Blondin

Bassin Maïpouri

Patagaïe

Manioc

Ouanary

Abris-sous-roche :

Abri Marcel

Abri Patagaïe

Carapa-II

Carapa-III

Carbet Mitan

Gaston

Moustique

Sébéloué-I

Sébéloué-II

Sébéloué-III

Sébéloué-IV

Trou Comou

Trou Kalina

Trou Pac

Trou Tigre

Funéraire :

Caripo

Gravier

Carapa-I

Enfer

Gros Montagne

Jarre Indien

Trou Agae

Trou Biche

Trou Coq de Roche

Trou Delft

Trou Reliquaire

Tessons intrusifs :

Baugé

Mapaou

Saut Mapaou

Crique Brémont

Glycérias

Thémire

Sainte Agathe
BARBAKOEBA

Crique Jacques

THEMIRE

Almaric

Anse Legoff

Bois Diable

Crique Brémont

Crique Météreau

Glycérias

Kamuyune

Karouabo

Léonard

Mini-Circuit Automobile

Mont La Calotte

Montravel

Pascaud

Route de Montabo

Sainte Agathe

Saramaca

Thémire

Vieux Chemin

\section{KORIABO}

Baugé

Coswine

Counamari

Crique Petit Approuague

Dubol

Grand Vevoni

Kormontibo

Mapaou

Piste de Papaichton

Piste Météo

P.K. 9,3

Route de Mana P.K. 26,5

Saut Mapaou

Tourépé

Tessons intrusifs :

Crique Brémont

Mini-Circuit Automobile

Pascaud

Sainte Agathe

Ti-Jarre 
ANNEXE 2 : DATATIONS AU ${ }^{14} \mathrm{C}$ OBTENUES DURANT LA RECHERCHE ${ }^{1}$

\begin{tabular}{|c|c|c|c|c|}
\hline site & code & datation ${ }^{14} \mathrm{C}$ & calibrage & probabilité \\
\hline \multicolumn{5}{|c|}{ Complexe Aristé } \\
\hline Carbet Mitan & OBDY 653 & $2070+$ ou $-45 \mathrm{BP}$ & $164-39 \mathrm{BC}$ & $100 \%$ \\
\hline Carbet Mitan & OBDY 650 & $1650+$ ou $-40 \mathrm{BP}$ & $345-427 \mathrm{AD}$ & $100 \%$ \\
\hline Abri Marcel & OBDY 798 & $1470+$ ou $-40 \mathrm{BP}$ & $551-637 \mathrm{AD}$ & $100 \%$ \\
\hline Abri Marcel & OBDY 799 & $1430+$ ou $-30 \mathrm{BP}$ & $598-645 \mathrm{AD}$ & $100 \%$ \\
\hline Abri Marcel & OBDY 795 & $1400+$ ou $-60 \mathrm{BP}$ & $580-671 \mathrm{AD}$ & $100 \%$ \\
\hline Abri Marcel & OBDY 797 & $1310+$ ou $-35 \mathrm{BP}$ & $\begin{array}{l}659-715 \text { AD } \\
745-767 \text { AD }\end{array}$ & $\begin{array}{l}71,4 \% \\
28,6 \%\end{array}$ \\
\hline Abri Marcel & OBDY 800 & $1170+$ ou $-30 \mathrm{BP}$ & $\begin{array}{l}781-791 \text { AD } \\
803-892 \text { AD } \\
928-933 \text { AD }\end{array}$ & $\begin{array}{r}9,8 \% \\
85,5 \% \\
4,7 \% \\
\end{array}$ \\
\hline Trou Agae & OBDY 732 & $310+$ ou $-110 \mathrm{BP}$ & $\begin{array}{l}1446-1669 \text { AD } \\
1778-1790 \text { AD }\end{array}$ & $\begin{array}{c}96,5 \% \\
3,5 \% \\
\end{array}$ \\
\hline \multicolumn{5}{|c|}{ Complexe Thémire } \\
\hline Bois Diable & OBDY 794 & $510+$ ou $-40 \mathrm{BP}$ & $\begin{array}{l}1335-1337 \text { AD } \\
1399-1435 \text { AD }\end{array}$ & $\begin{array}{r}5,2 \% \\
94,8 \% \\
\end{array}$ \\
\hline Sainte Agathe & OBDY 796 & $380+$ ou $-35 \mathrm{BP}$ & $\begin{array}{l}1444-1514 \text { AD } \\
1601-1616 \text { AD }\end{array}$ & $\begin{array}{l}84,7 \% \\
15,3 \% \\
\end{array}$ \\
\hline Glycérias & OBDY 727 & $260+$ ou $-50 \mathrm{BP}$ & $\begin{array}{l}1518-1588 \mathrm{AD} \\
1622-1670 \mathrm{AD} \\
1773-1792 \mathrm{AD} \\
1947-1948 \mathrm{AD}\end{array}$ & $\begin{array}{c}46,5 \% \\
42,3 \% \\
10,2 \% \\
0,9 \%\end{array}$ \\
\hline Glycérias & OBDY 717 & $80+$ ou $-70 \mathrm{BP}$ & $\begin{array}{l}1691-1732 \mathrm{AD} \\
1812-1926 \mathrm{AD}\end{array}$ & $\begin{array}{l}26 \% \\
74 \%\end{array}$ \\
\hline Rte de Montabo & OBDY 523 & $170+$ ou $-170 \mathrm{BP}$ & $1642-1950 \mathrm{AD}$ & $100 \%$ \\
\hline \multicolumn{5}{|c|}{ Complexe Koriabo } \\
\hline Mapaou & OBDY 728 & $240+$ ou $-50 \mathrm{BP}$ & $\begin{array}{l}1526-1561 \text { AD } \\
1629-1678 \text { AD } \\
1744-1801 \text { AD } \\
1939-1953 \text { AD }\end{array}$ & $\begin{array}{c}16,3 \% \\
39,7 \% \\
36,2 \% \\
7,8 \%\end{array}$ \\
\hline
\end{tabular}

1. Les datations au ${ }^{14} \mathrm{C}$ ont été réalisées à l'unité de Géochimie Isotopique du laboratoire des Formations Superficielles au centre ORSTOM de Bondy. Tous les âges disponibles ont été calibrés en utilisant une courbe continentale avec le programme de calibrage CalibETH 1.5b (AMS Facility, ETH Hönggeberg, Institute for Intermediate Energy Physics, ETH Zürich, Zwitzerland, 1991), la courbe étant sur le fichier ATM20.14B (Linik, Suess, Becker, Stuiver, Kromer, Ferguson, Rhein, Schoch, Munnich, Long, Damon, « Average Dataset of LSB, SKBF, KRBSMSB/20 years steps. Radiocarbon, 28 (2B), 1986, 805-1 021). Les mesures retenues sont à un sigma. Dans certains cas, plusieurs fourchettes de temps sont proposées par le calibrage. Chacune d'entre elles a la même probabilité d'être juste et une fourchette peut alors être choisie en fonction des données archéologiques. 